\title{
Molecular Vibrations Induced Potential Diradical Character in Hexazapentacene
}

Yiwei Feng, Fengying Zhang, Xinyu Song, Yuxiang Bu*

School of Chemistry and Chemical Engineering, Shandong University, Jinan, 250100, P. R. China

\section{Supporting Information}

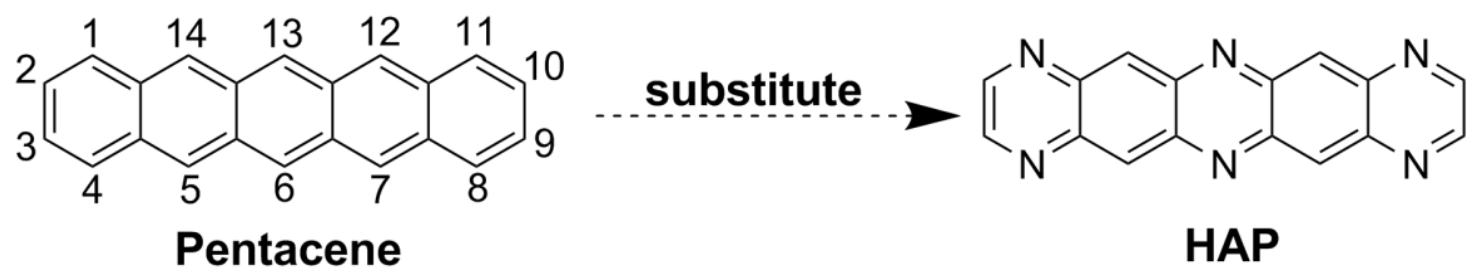

Figure S1. The structures of pentacene and HAP

Table S1. Energy orders of 19 distorted configurations induced by 19 diradical character of positive distortion, calculated at the UB3LYP/6-31++G(d,p) level

\begin{tabular}{ccccc}
\hline Mode & $\mathrm{E}_{(\mathrm{CS})}$ & $\mathrm{E}_{(\mathrm{OS})}$ & $\mathrm{E}_{(\mathrm{OT})}$ & $\mathrm{E}_{1} r(\mathrm{gy})$ \\
\hline 17 & -942.8719038 & -942.8726627 & -942.8496679 & $\mathrm{E}_{(\mathrm{OS})}<\mathrm{E}_{(\mathrm{CS})}<\mathrm{E}_{(\mathrm{OT})}$ \\
19 & -942.7927868 & -942.7941513 & -942.7689859 & $\mathrm{E}_{(\mathrm{OS})}<\mathrm{E}_{(\mathrm{CS})}<\mathrm{E}_{(\mathrm{OT})}$ \\
25 & -942.7615054 & -942.7675150 & -942.7762433 & $\mathrm{E}_{(\mathrm{OT})}<\mathrm{E}_{(\mathrm{OS})}<\mathrm{E}_{(\mathrm{CS})}$ \\
27 & -942.8489905 & -942.8490472 & -942.8214497 & $\mathrm{E}_{(\mathrm{OS})}<\mathrm{E}_{(\mathrm{CS})}<\mathrm{E}_{(\mathrm{OT})}$ \\
29 & -942.5946466 & -942.5965634 & -942.5793692 & $\mathrm{E}_{(\mathrm{OS})}<\mathrm{E}_{(\mathrm{CS})}<\mathrm{E}_{(\mathrm{OT})}$ \\
34 & -942.6398977 & -942.6509197 & -942.6421976 & $\mathrm{E}_{(\mathrm{OS})}<\mathrm{E}_{(\mathrm{OT})}<\mathrm{E}_{(\mathrm{CS})}$ \\
48 & -942.7127946 & -942.7145716 & -942.6965517 & $\mathrm{E}_{(\mathrm{OS})}<\mathrm{E}_{(\mathrm{CS})}<\mathrm{E}_{(\mathrm{OT})}$ \\
58 & -942.7248613 & -942.7254930 & -942.6975455 & $\mathrm{E}_{(\mathrm{OS})}<\mathrm{E}_{(\mathrm{CS})}<\mathrm{E}_{(\mathrm{OT})}$ \\
60 & -942.4891061 & -942.5063535 & -942.5131118 & $\mathrm{E}_{(\mathrm{OT})}<\mathrm{E}_{(\mathrm{OS})}<\mathrm{E}_{(\mathrm{CS})}$ \\
62 & -941.1497526 & -941.1546133 & -941.1599238 & $\mathrm{E}_{(\mathrm{OT})}<\mathrm{E}_{(\mathrm{OS})}<\mathrm{E}_{(\mathrm{CS})}$ \\
64 & -942.7713039 & -942.7713041 & -942.7401290 & $\mathrm{E}_{(\mathrm{OS})}<\mathrm{E}_{(\mathrm{CS})}<\mathrm{E}_{(\mathrm{OT})}$ \\
65 & -942.4867245 & -942.4888407 & -942.4915897 & $\mathrm{E}_{(\mathrm{OT})}<\mathrm{E}_{(\mathrm{OS})}<\mathrm{E}_{(\mathrm{CS})}$ \\
66 & -942.4859355 & -942.4959130 & -942.4858357 & $\mathrm{E}_{(\mathrm{OS})}<\mathrm{E}_{(\mathrm{CS})}<\mathrm{E}_{(\mathrm{OT})}$ \\
68 & -940.7927029 & -940.8279255 & -940.8251939 & $\mathrm{E}_{(\mathrm{OS})}<\mathrm{E}_{(\mathrm{OT})}<\mathrm{E}_{(\mathrm{CS})}$ \\
71 & -941.5813690 & -941.5916056 & -941.5913690 & $\mathrm{E}_{(\mathrm{OS})}<\mathrm{E}_{(\mathrm{OT})}<\mathrm{E}_{(\mathrm{CS})}$ \\
72 & -941.9400969 & -941.9917553 & -941.9428350 & $\mathrm{E}_{(\mathrm{OS})}<\mathrm{E}_{(\mathrm{OT})}<\mathrm{E}_{(\mathrm{CS})}$ \\
73 & -941.1376754 & -941.1718978 & -941.1703192 & $\mathrm{E}_{(\mathrm{OS})}<\mathrm{E}_{(\mathrm{OT})}<\mathrm{E}_{(\mathrm{CS})}$ \\
74 & -941.1500031 & -941.1587406 & -941.1625902 & $\mathrm{E}_{(\mathrm{OT})}<\mathrm{E}_{(\mathrm{OS})}<\mathrm{E}_{(\mathrm{CS})}$ \\
75 & -941.2206607 & -941.2208473 & -941.2086400 & $\mathrm{E}_{(\mathrm{OS})}<\mathrm{E}_{(\mathrm{CS})}<\mathrm{E}_{(\mathrm{OT})}$ \\
\hline
\end{tabular}


Table S2. Energy orders of 65 distorted configurations according to nondiradical character of positive distortion, calculated at the UB3LYP/6-31++G(d,p) level

\begin{tabular}{|c|c|c|c|}
\hline Mode & $\mathrm{E}_{(\mathrm{CS})}$ & $\mathrm{E}_{(\mathrm{OT})}$ & Energy Order \\
\hline 1 & -943.0576279 & -943.0167367 & $\mathrm{E}_{(\mathrm{CS})}<\mathrm{E}_{(\mathrm{OT})}$ \\
\hline 2 & -943.0553172 & -943.0110122 & $\mathrm{E}_{(\mathrm{CS})}<\mathrm{E}_{(\mathrm{OT})}$ \\
\hline 3 & -943.0531103 & -943.0130077 & $\mathrm{E}_{(\mathrm{CS})}<\mathrm{E}_{(\mathrm{OT})}$ \\
\hline 4 & -943.0502607 & -943.0081529 & $\mathrm{E}_{(\mathrm{CS})}<\mathrm{E}_{(\mathrm{OT})}$ \\
\hline 5 & -943.0472642 & -943.0046993 & $\mathrm{E}_{(\mathrm{CS})}<\mathrm{E}_{(\mathrm{OT})}$ \\
\hline 6 & -943.0350625 & -942.9981799 & $\mathrm{E}_{(\mathrm{CS})}<\mathrm{E}_{(\mathrm{OT})}$ \\
\hline 7 & -943.0426834 & -942.9999152 & $\mathrm{E}_{(\mathrm{CS})}<\mathrm{E}_{(\mathrm{OT})}$ \\
\hline 8 & -943.0209066 & -942.9781925 & $\mathrm{E}_{(\mathrm{CS})}<\mathrm{E}_{(\mathrm{OT})}$ \\
\hline 9 & -943.0170051 & -942.9830970 & $\mathrm{E}_{(\mathrm{CS})}<\mathrm{E}_{(\mathrm{OT})}$ \\
\hline 10 & -943.0050109 & -942.9709884 & $\mathrm{E}_{(\mathrm{CS})}<\mathrm{E}_{(\mathrm{OT})}$ \\
\hline 11 & -943.0091655 & -942.9725902 & $\mathrm{E}_{(\mathrm{CS})}<\mathrm{E}_{(\mathrm{OT})}$ \\
\hline 12 & -942.9941060 & -942.9522327 & $\mathrm{E}_{(\mathrm{CS})}<\mathrm{E}_{(\mathrm{OT})}$ \\
\hline 13 & -942.9905521 & -942.9587546 & $\mathrm{E}_{(\mathrm{CS})}<\mathrm{E}_{(\mathrm{OT})}$ \\
\hline 14 & -942.9155132 & -942.8801320 & $\mathrm{E}_{(\mathrm{CS})}<\mathrm{E}_{(\mathrm{OT})}$ \\
\hline 15 & -942.9923603 & -942.9583435 & $\mathrm{E}_{(\mathrm{CS})}<\mathrm{E}_{(\mathrm{OT})}$ \\
\hline 16 & -943.0033198 & -942.9661413 & $\mathrm{E}_{(\mathrm{CS})}<\mathrm{E}_{(\mathrm{OT})}$ \\
\hline 18 & -942.8908690 & -942.8529495 & $\mathrm{E}_{(\mathrm{CS})}<\mathrm{E}_{(\mathrm{OT})}$ \\
\hline 20 & -942.9755553 & -942.9360149 & $\mathrm{E}_{(\mathrm{CS})}<\mathrm{E}_{(\mathrm{OT})}$ \\
\hline 21 & -942.9753922 & -942.9407905 & $\mathrm{E}_{(\mathrm{CS})}<\mathrm{E}_{(\mathrm{OT})}$ \\
\hline 22 & -942.9467260 & -942.9078206 & $\mathrm{E}_{(\mathrm{CS})}<\mathrm{E}_{(\mathrm{OT})}$ \\
\hline 23 & -942.7933844 & -942.7369834 & $\mathrm{E}_{(\mathrm{CS})}<\mathrm{E}_{(\mathrm{OT})}$ \\
\hline 24 & -942.7935353 & -942.7597935 & $\mathrm{E}_{(\mathrm{CS})}<\mathrm{E}_{(\mathrm{OT})}$ \\
\hline 26 & -942.8723330 & -942.8246115 & $\mathrm{E}_{(\mathrm{CS})}<\mathrm{E}_{(\mathrm{OT})}$ \\
\hline 28 & -942.7312401 & -942.6844797 & $\mathrm{E}_{(\mathrm{CS})}<\mathrm{E}_{(\mathrm{OT})}$ \\
\hline 30 & -942.7290418 & -942.6858914 & $\mathrm{E}_{(\mathrm{CS})}<\mathrm{E}_{(\mathrm{OT})}$ \\
\hline 31 & -942.9170643 & -942.8778903 & $\mathrm{E}_{(\mathrm{CS})}<\mathrm{E}_{(\mathrm{OT})}$ \\
\hline 32 & -942.8820888 & -942.8427059 & $\mathrm{E}_{(\mathrm{CS})}<\mathrm{E}_{(\mathrm{OT})}$ \\
\hline 33 & -942.9091428 & -942.8556815 & $\mathrm{E}_{(\mathrm{CS})}<\mathrm{E}_{(\mathrm{OT})}$ \\
\hline 35 & -942.7593837 & -942.7437703 & $\mathrm{E}_{(\mathrm{CS})}<\mathrm{E}_{(\mathrm{OT})}$ \\
\hline 36 & -942.9800540 & -942.9382606 & $\mathrm{E}_{(\mathrm{CS})}<\mathrm{E}_{(\mathrm{OT})}$ \\
\hline 37 & -942.9791348 & -942.9377927 & $\mathrm{E}_{(\mathrm{CS})}<\mathrm{E}_{(\mathrm{OT})}$ \\
\hline 38 & -942.9394954 & -942.9020619 & $\mathrm{E}_{(\mathrm{CS})}<\mathrm{E}_{(\mathrm{OT})}$ \\
\hline 39 & -942.9357309 & -942.9000042 & $\mathrm{E}_{(\mathrm{CS})}<\mathrm{E}_{(\mathrm{OT})}$ \\
\hline 40 & -942.9130641 & -942.8724651 & $\mathrm{E}_{(\mathrm{CS})}<\mathrm{E}_{(\mathrm{OT})}$ \\
\hline 41 & -942.9624196 & -942.9234579 & $\mathrm{E}_{(\mathrm{CS})}<\mathrm{E}_{(\mathrm{OT})}$ \\
\hline 42 & -942.9529741 & -942.9147772 & $\mathrm{E}_{(\mathrm{CS})}<\mathrm{E}_{(\mathrm{OT})}$ \\
\hline 43 & -942.6611821 & -942.6188949 & $\mathrm{E}_{(\mathrm{CS})}<\mathrm{E}_{(\mathrm{OT})}$ \\
\hline 44 & -942.6636529 & -942.6309518 & $\mathrm{E}_{(\mathrm{CS})}<\mathrm{E}_{(\mathrm{OT})}$ \\
\hline 45 & -942.9350031 & -942.8953893 & $\mathrm{E}_{(\mathrm{CS})}<\mathrm{E}_{(\mathrm{OT})}$ \\
\hline 46 & -942.9348143 & -942.8952327 & $\mathrm{E}_{(\mathrm{CS})}<\mathrm{E}_{(\mathrm{OT})}$ \\
\hline
\end{tabular}




\begin{tabular}{llll}
\hline 47 & -942.8026022 & -942.7576203 & $\mathrm{E}_{(\mathrm{CS})}<\mathrm{E}_{(\mathrm{OT})}$ \\
49 & -942.5939058 & -942.4885343 & $\mathrm{E}_{(\mathrm{CS})}<\mathrm{E}_{(\mathrm{OT})}$ \\
50 & -942.7519707 & -942.7138688 & $\mathrm{E}_{(\mathrm{CS})}<\mathrm{E}_{(\mathrm{OT})}$ \\
51 & -942.8941129 & -942.8287069 & $\mathrm{E}_{(\mathrm{CS})}<\mathrm{E}_{(\mathrm{OT})}$ \\
52 & -942.8081881 & -942.7717031 & $\mathrm{E}_{(\mathrm{CS})}<\mathrm{E}_{(\mathrm{OT})}$ \\
53 & -942.8688531 & -942.8287069 & $\mathrm{E}_{(\mathrm{CS})}<\mathrm{E}_{(\mathrm{OT})}$ \\
54 & -942.8915268 & -942.8496582 & $\mathrm{E}_{(\mathrm{CS})}<\mathrm{E}_{(\mathrm{OT})}$ \\
55 & -942.6402170 & -942.5892471 & $\mathrm{E}_{(\mathrm{CS})}<\mathrm{E}_{(\mathrm{OT})}$ \\
56 & -942.3423950 & -942.3254200 & $\mathrm{E}_{(\mathrm{CS})}<\mathrm{E}_{(\mathrm{OT})}$ \\
57 & -942.4056255 & -942.3713378 & $\mathrm{E}_{(\mathrm{CS})}<\mathrm{E}_{(\mathrm{OT})}$ \\
59 & -942.7641883 & -942.7284243 & $\mathrm{E}_{(\mathrm{CS})}<\mathrm{E}_{(\mathrm{OT})}$ \\
61 & -942.8354077 & -942.7963397 & $\mathrm{E}_{(\mathrm{CS})}<\mathrm{E}_{(\mathrm{OT})}$ \\
63 & -942.8403215 & -942.8005476 & $\mathrm{E}_{(\mathrm{CS})}<\mathrm{E}_{(\mathrm{OT})}$ \\
67 & -941.6994065 & -941.6752803 & $\mathrm{E}_{(\mathrm{CS})}<\mathrm{E}_{(\mathrm{OT})}$ \\
69 & -940.2289571 & -940.2276386 & $\mathrm{E}_{(\mathrm{CS})}<\mathrm{E}_{(\mathrm{OT})}$ \\
70 & -941.6860804 & -941.6520384 & $\mathrm{E}_{(\mathrm{CS})}<\mathrm{E}_{(\mathrm{OT})}$ \\
76 & -941.3593969 & -941.3561149 & $\mathrm{E}_{(\mathrm{CS})}<\mathrm{E}_{(\mathrm{OT})}$ \\
77 & -940.0188773 & -939.9775491 & $\mathrm{E}_{(\mathrm{CS})}<\mathrm{E}_{(\mathrm{OT})}$ \\
78 & -940.0181554 & -939.9768296 & $\mathrm{E}_{(\mathrm{CS})}<\mathrm{E}_{(\mathrm{OT})}$ \\
79 & -942.8204588 & -942.7778921 & $\mathrm{E}_{(\mathrm{CS})}<\mathrm{E}_{(\mathrm{OT})}$ \\
80 & -942.8237833 & -942.7816710 & $\mathrm{E}_{(\mathrm{CS})}<\mathrm{E}_{(\mathrm{OT})}$ \\
81 & -941.4767252 & -941.4347136 & $\mathrm{E}_{(\mathrm{CS})}<\mathrm{E}_{(\mathrm{OT})}$ \\
82 & -941.4765968 & -941.4338256 & $\mathrm{E}_{(\mathrm{CS})}<\mathrm{E}_{(\mathrm{OT})}$ \\
83 & -941.2584802 & -941.2166178 & $\mathrm{E}_{(\mathrm{CS})}<\mathrm{E}_{(\mathrm{OT})}$ \\
84 & -940.1870131 & -940.1452518 & $\mathrm{E}_{(\mathrm{CS})}<\mathrm{E}_{(\mathrm{OT})}$ \\
\hline
\end{tabular}

Table S3. Calculated energies (in au), $\left\langle\mathrm{S}^{2}\right\rangle$ values, and intramolecular magnetic exchange coupling constants $\left(J\right.$ in $\left.\mathrm{cm}^{-1}\right)$ at the UB3LYP/6-31++G(d,p) level for all distorted configurations with diradical character of positive distortion.

\begin{tabular}{cccc}
\hline Vibrational Modes & $\mathbf{E}_{(\mathbf{T})}\left(\left\langle\mathbf{S}^{2}\right\rangle\right)$ & $\mathbf{E}_{(\mathbf{B S})}\left(\left\langle\mathbf{S}^{2}\right\rangle\right)$ & $\boldsymbol{J}\left(\mathbf{c m}^{-\mathbf{1}}\right)$ \\
\hline 17 & $-942.84966790(2.040)$ & $-942.87266270(0.381)$ & -3041.7 \\
19 & $-942.76898590(2.042)$ & $-942.79415130(0.454)$ & -3479.5 \\
25 & $-942.77624330(2.017)$ & $-942.76751500(0.728)$ & 1485.4 \\
27 & $-942.82144970(2.080)$ & $-942.84904720(0.115)$ & -3081.8 \\
29 & $-942.57936920(2.016)$ & $-942.59656340(0.559)$ & -2590.6 \\
34 & $-942.64219760(2.023)$ & $-942.65091970(1.061)$ & -1991.4 \\
48 & $-942.69655170(2.038)$ & $-942.71457160(0.534)$ & -2631.2 \\
58 & $-942.69754550(2.022)$ & $-942.72549300(0.272)$ & -3505.6 \\
60 & $-942.51311180(2.018)$ & $-942.50635350(0.931)$ & 1364.1 \\
62 & $-941.15992380(2.014)$ & $-941.15461330(0.867)$ & 1016.5 \\
64 & $-942.74012900(2.027)$ & $-942.77130410(0.006)$ & -3386.8
\end{tabular}




\begin{tabular}{llll}
65 & $-942.49158970(2.016)$ & $-942.48884070(0.602)$ & 426.7 \\
66 & $-942.48583570(2.028)$ & $-942.49591300(0.853)$ & -1881.2 \\
68 & $-940.82519390(2.017)$ & $-940.82792550(1.034)$ & -609.9 \\
71 & $-941.59136900(2.585)$ & $-941.59160560(1.593)$ & -52.4 \\
72 & $-941.94283500(2.141)$ & $-941.99175530(1.868)$ & -39388.1 \\
73 & $-941.17031920(2.026)$ & $-941.17189780(1.077)$ & -365.2 \\
74 & $-941.16259020(2.730)$ & $-941.15874060(1.278)$ & 581.9 \\
75 & $-941.20864000(2.133)$ & $-941.22084730(0.160)$ & -1357.7 \\
\hline
\end{tabular}

Note: We use a simple broken-symmetry (BS) formalism which was developed by Yamaguchi et al. and regarded as the most appropriate one for estimating magnetic exchange coupling interaction in diradicals to estimate the $J$ value. The formula is given as $J=$ $\frac{E_{B S}-E_{T}}{\left\langle S^{2}\right\rangle_{T}-\left\langle S^{2}\right\rangle_{B S}}$, where $E_{B S}$ and $E_{T}$ refer to the energies of unrestricted open-shell BS singlet and triplet states, while $\left\langle\mathrm{S}^{2}\right\rangle_{\mathrm{BS}}$ and $\left\langle\mathrm{S}^{2}\right\rangle_{\mathrm{T}}$ are the corresponding average spin square $\left\langle\mathrm{S}^{2}>\right.$ values, respectively.

Table S4. 84 vibrational modes HAP of positive distortion and the corresponding geometrical characters, HOMO-LUMO gaps (H-L gap, eV) of the closed-shell singlet states, T-S gaps between the closed-shell singlet and triplet states (E(triplet)-E(singlet), $\mathrm{kcal} / \mathrm{mol})$, and $\left\langle\mathrm{S}^{2}\right\rangle$ values, SOMO $(\alpha, \beta)$, spin densities of the OS states of the mode-distorted HAP configurations. Note that the distorted configuration denotes its largest distortion for each vibrational mode.

\begin{tabular}{|c|c|c|c|c|c|}
\hline Modes/ $\mathbf{S}^{2}$ & $\begin{array}{c}\text { HO-LU } \\
\text { gap } \\
\text { (ev) } \\
\text { T-S gap } \\
\text { (kcal/mol } \\
\text { ) }\end{array}$ & Distortion Modes & $\begin{array}{l}\text { HOMO or SOMO } \\
(\alpha)\end{array}$ & $\begin{array}{c}\text { LUMO or SOMO } \\
(\beta)\end{array}$ & Spin density \\
\hline $\begin{array}{l}1 \\
0.00\end{array}$ & $\begin{array}{c}2.32 \\
25.61\end{array}$ & $020=000$ & & & \\
\hline $\begin{array}{l}2 \\
0.00\end{array}$ & $\begin{array}{l}2.40 \\
27.75\end{array}$ & $80828=20,9-3899$ & & & \\
\hline $\begin{array}{l}3 \\
0.00\end{array}$ & $\begin{array}{c}2.30 \\
25.12\end{array}$ & 000303000000 & & & \\
\hline
\end{tabular}




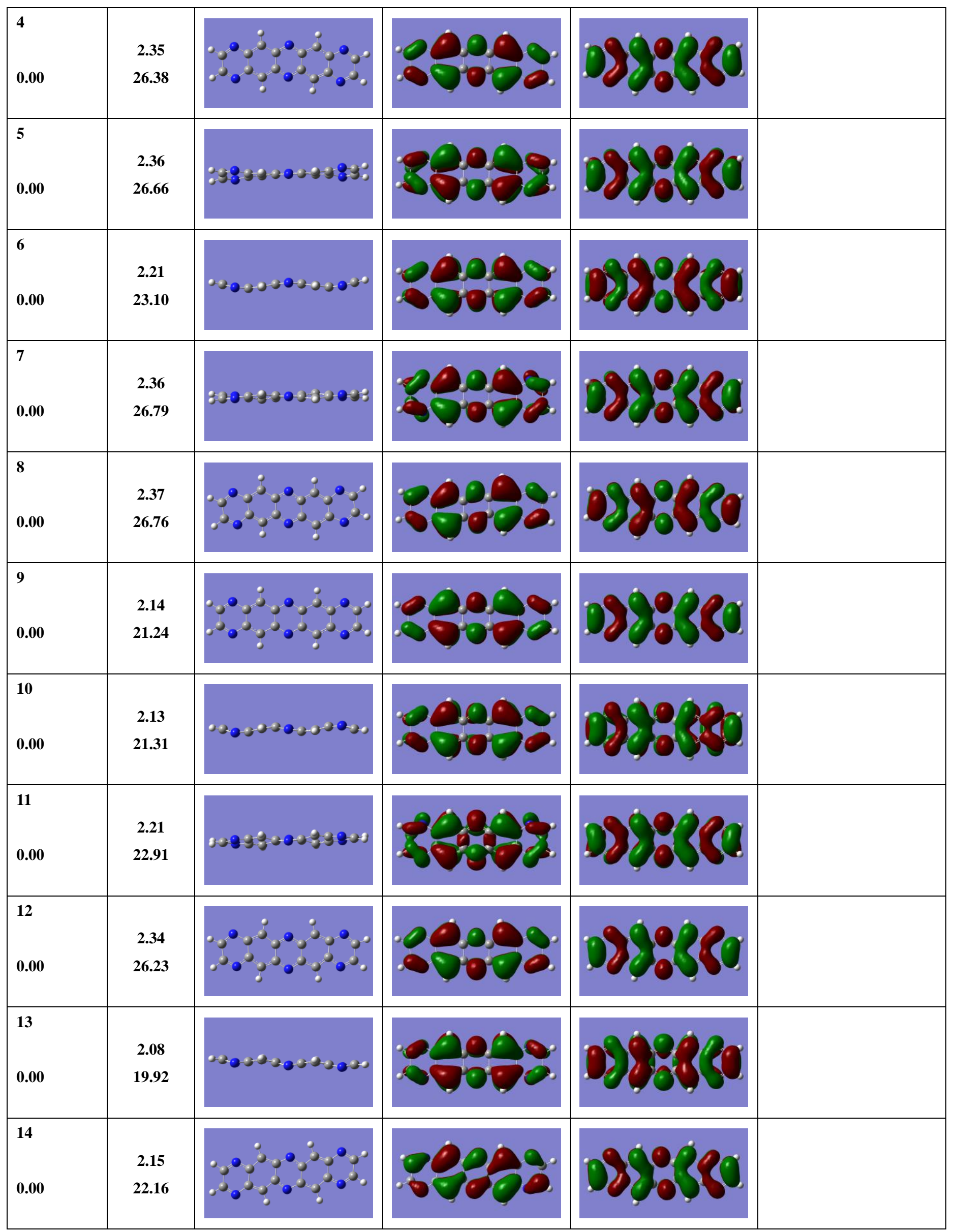




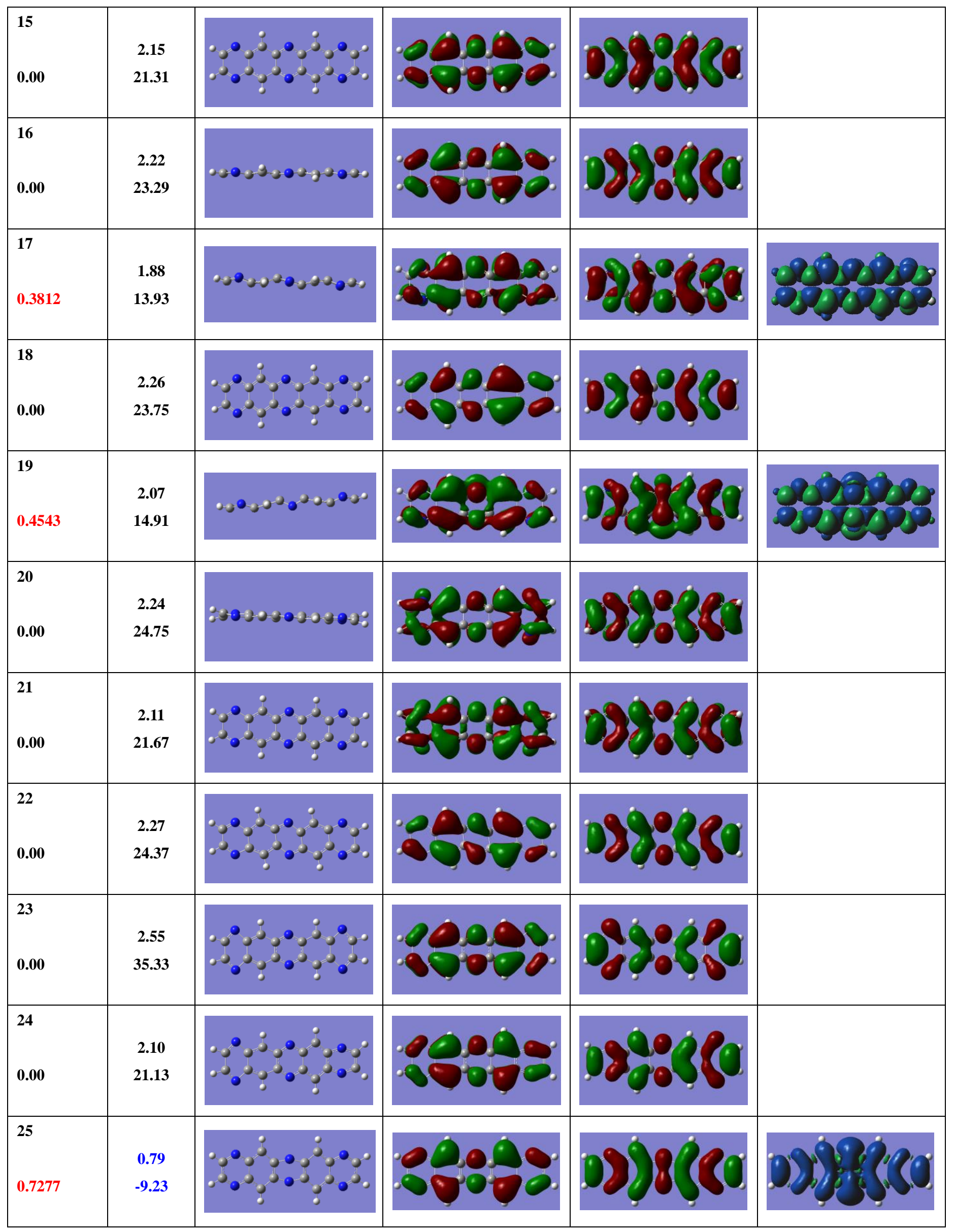




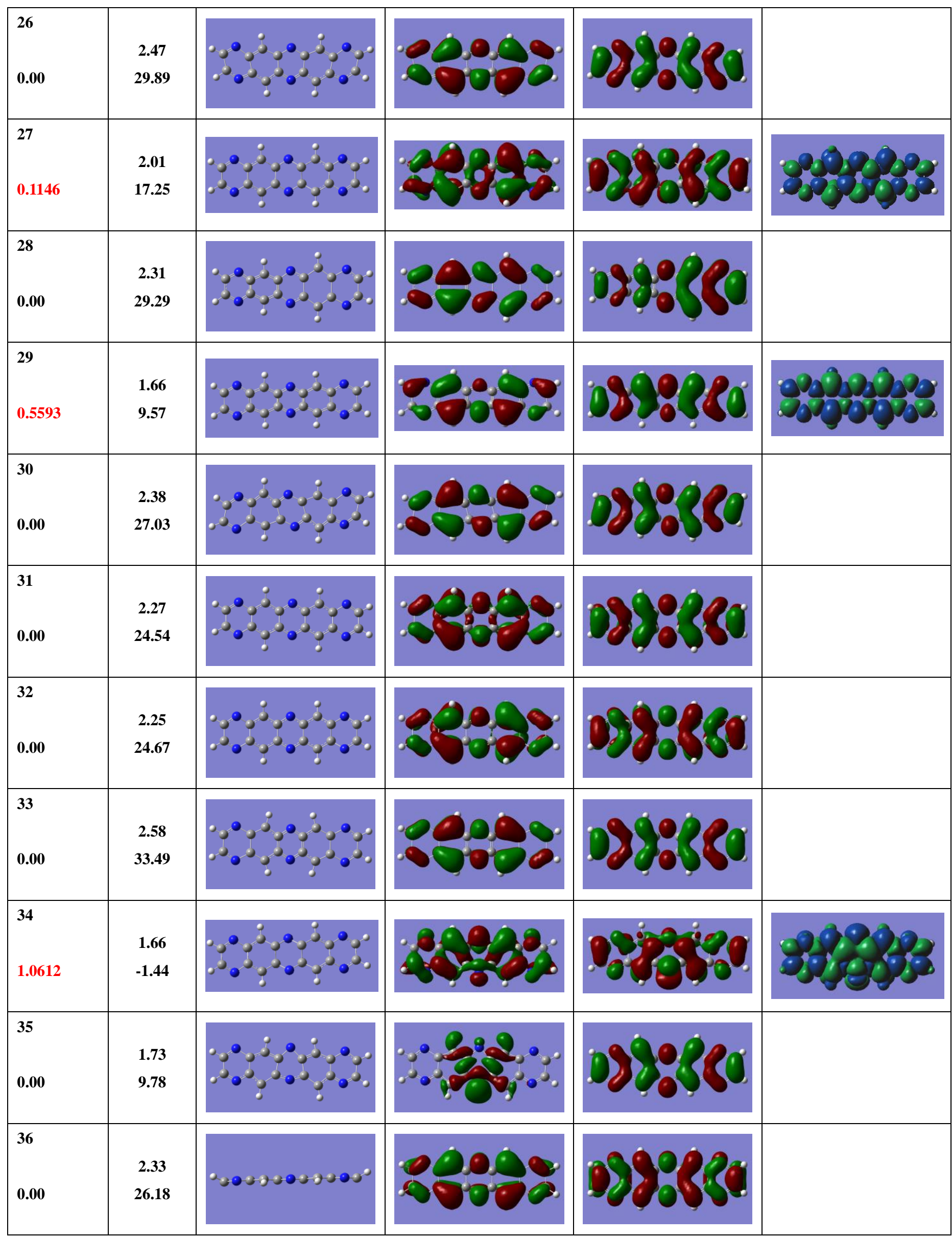




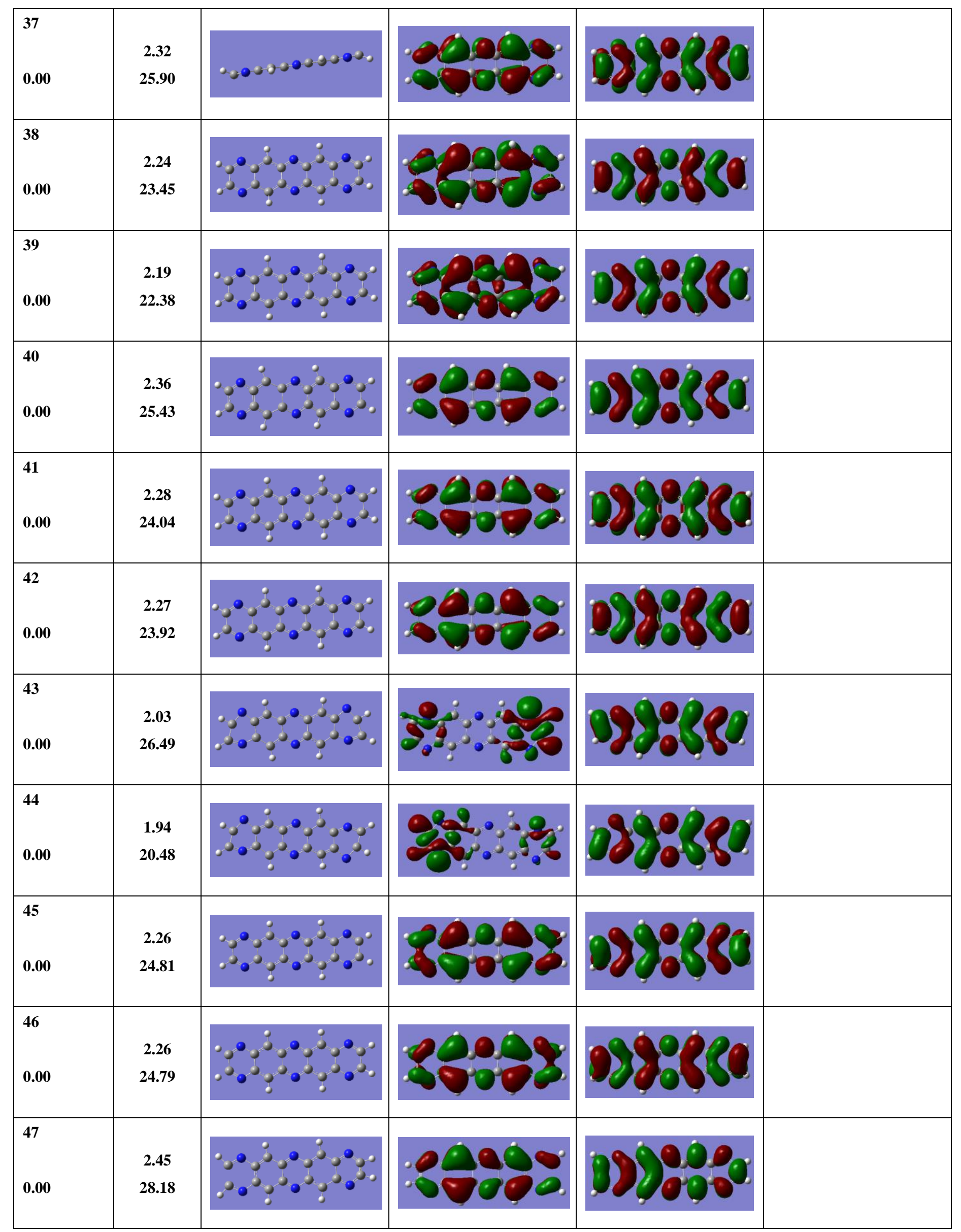




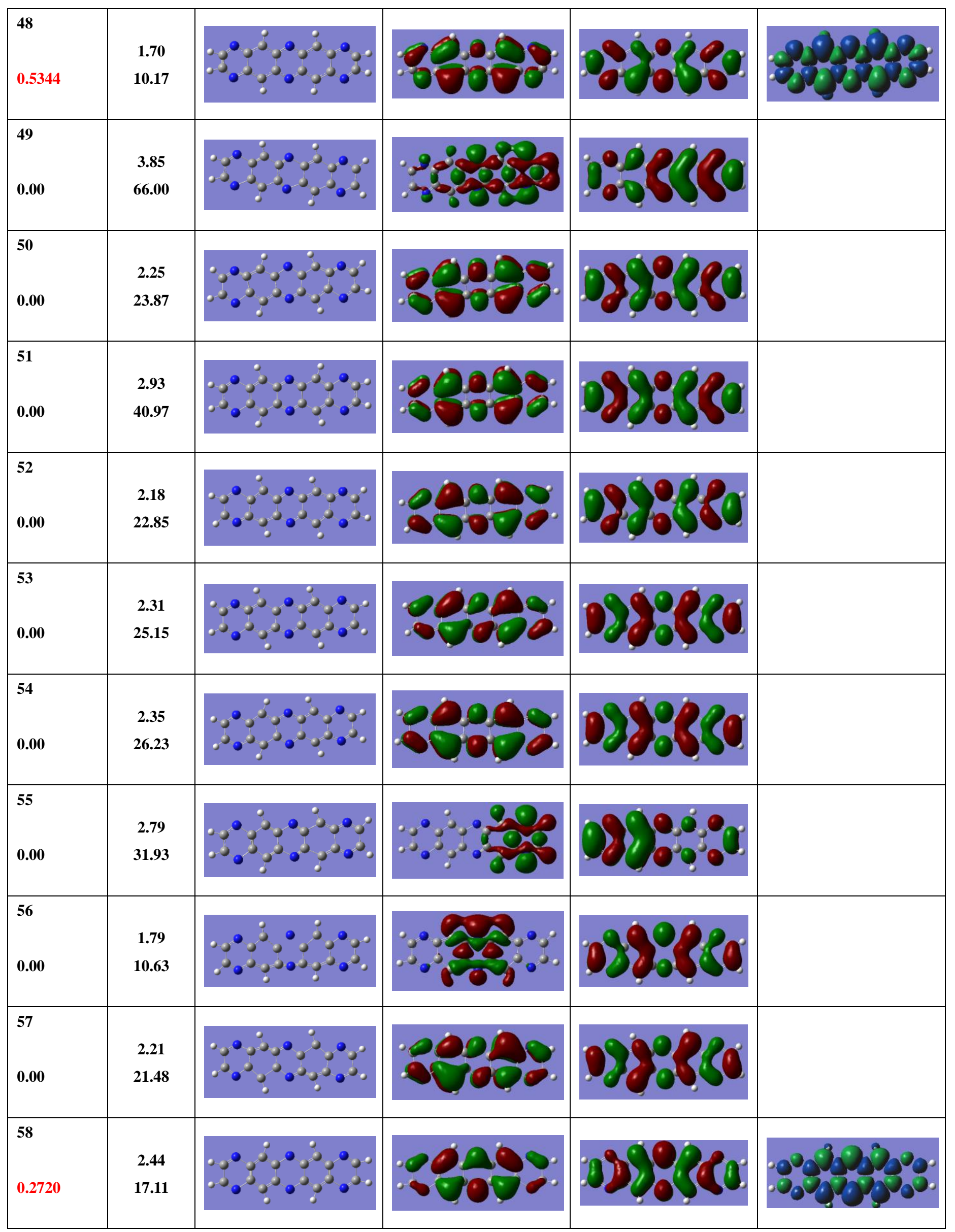




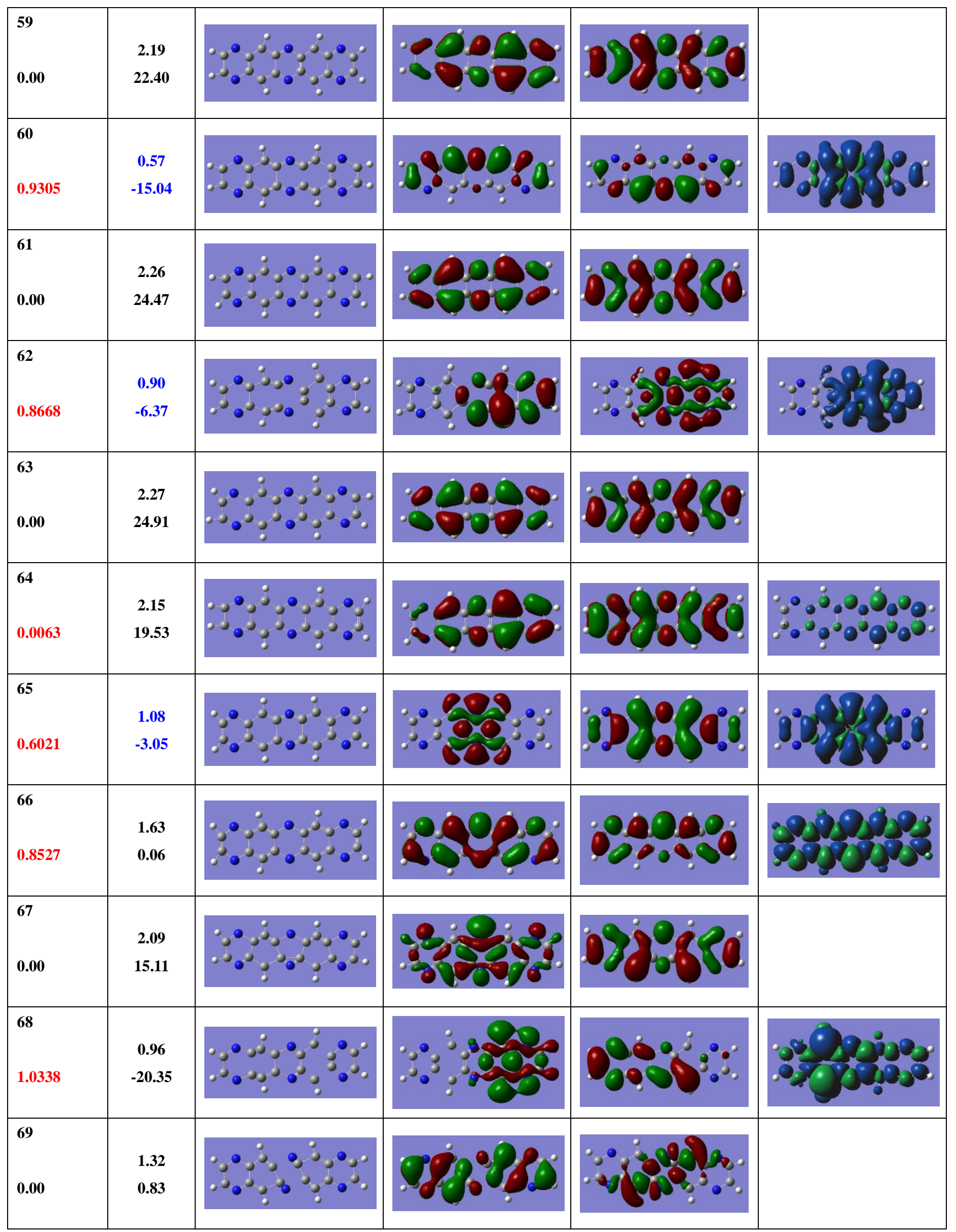




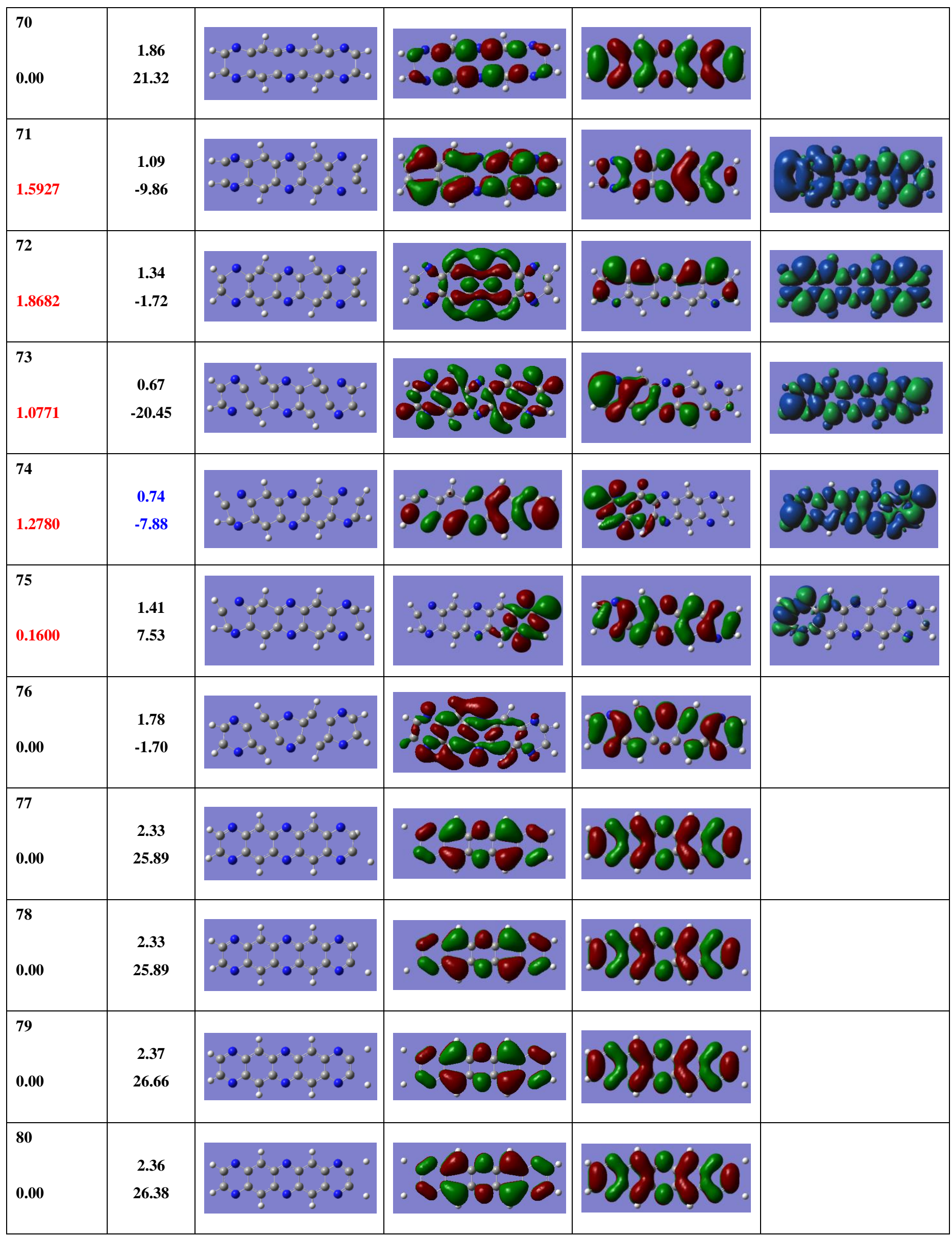




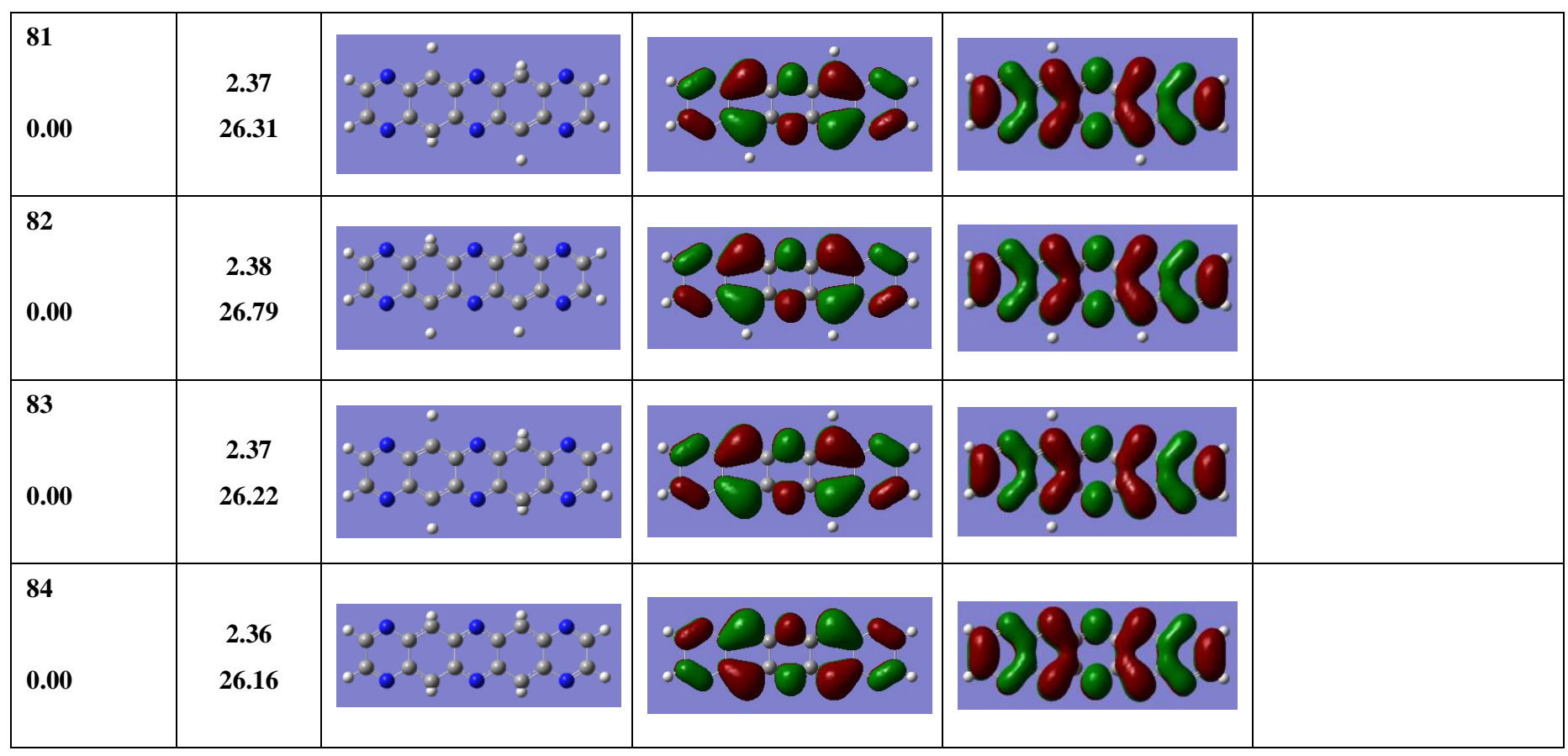

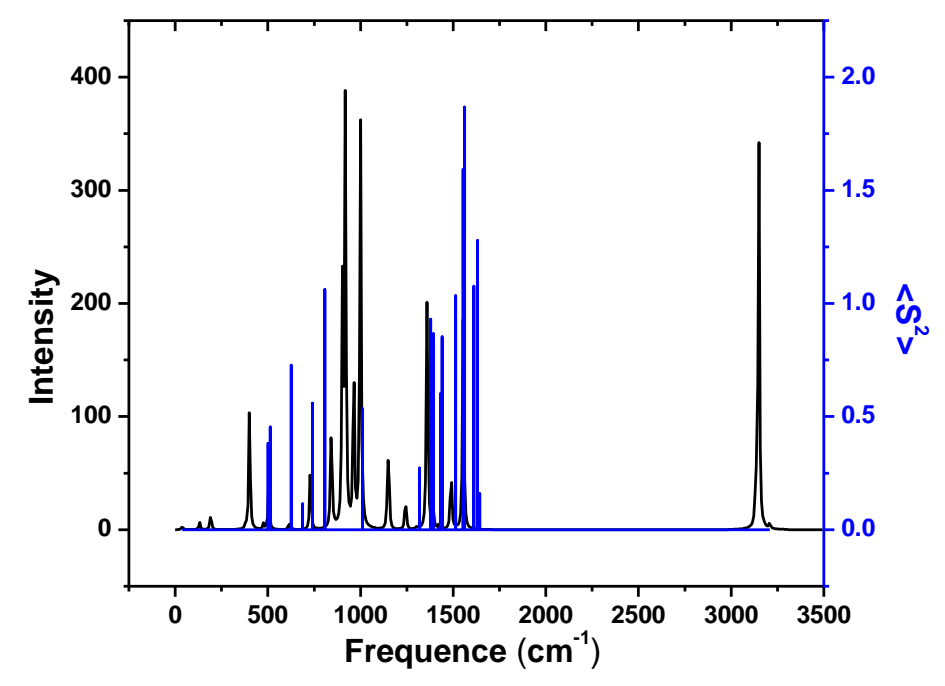

Figure S3. 84 normal modes of vibration of positive distortion which distributes in the region of $38.33-3208.66 \mathrm{~cm}^{-1}$ in HAP.

Table S5. Calculated energies (in au), $\left\langle\mathrm{S}^{2}\right\rangle$ values, and intramolecular magnetic exchange coupling constants $\left(J\right.$ in $\left.\mathrm{cm}^{-1}\right)$ at the UB3LYP/6-31++G(d,p) level for all decile distorted configurations with diradical character of positive distortion.

\begin{tabular}{cccc}
\hline Vibrational Modes & $\mathbf{E}_{(\mathbf{T})}\left(\left\langle\mathbf{S}^{\mathbf{2}}\right\rangle\right)$ & $\mathbf{E}_{(\mathbf{B S})}\left(\left\langle\mathbf{S}^{2}\right\rangle\right)$ & $\boldsymbol{J}\left(\mathbf{c m}^{-\mathbf{1}}\right)$ \\
\hline $17-8$ & $-942.91380000(2.036)$ & $-942.94270990(0.027)$ & -3159.4 \\
$17-9$ & $-942.88397670(2.038)$ & $-942.90989390(0.204)$ & -3102.5 \\
17 & $-942.84966790(2.040)$ & $-942.87266270(0.381)$ & -3041.7
\end{tabular}




\begin{tabular}{|c|c|c|c|}
\hline $19-8$ & $-942.86555520(2.038)$ & $-942.89738240(0.027)$ & -3473.5 \\
\hline $19-9$ & $-942.82057470(2.040)$ & $-942.84915850(0.242)$ & -3489.1 \\
\hline 19 & $-942.76898590(2.042)$ & $-942.79415130(0.454)$ & -3479.5 \\
\hline $25-9$ & $-942.81347940(2.017)$ & $-942.82333770(0.062)$ & 1106.7 \\
\hline 25 & $-942.77624330(2.017)$ & $-942.76751500(0.728)$ & 1485.4 \\
\hline 27 & $-942.82144970(2.080)$ & $-942.84904720(0.115)$ & -3081.8 \\
\hline $29-7$ & $-942.83181410(2.019)$ & $-942.85822770(0.064)$ & -2965.3 \\
\hline $29-8$ & $-942.76328870(2.018)$ & $-942.78660510(0.228)$ & -2858.9 \\
\hline $29-9$ & $-942.67983400(2.017)$ & $-942.70005030(0.395)$ & -2735.5 \\
\hline 29 & $-942.57936920(2.016)$ & $-942.59656340(0.559)$ & -2590.6 \\
\hline $34-6$ & $-942.87467530(2.033)$ & $-942.90384420(0.028)$ & -3192.9 \\
\hline $34-7$ & $-942.82560060(2.027)$ & $-942.84920220(0.320)$ & -3034.5 \\
\hline $34-8$ & $-942.77063930(2.021)$ & $-942.78825840(0.606)$ & -2732.8 \\
\hline $34-9$ & $-942.70955670(2.020)$ & $-942.72194440(0.856)$ & -2335.7 \\
\hline 34 & $-942.64219760(2.023)$ & $-942.65091970(1.061)$ & -1991.4 \\
\hline $48-6$ & $-942.93309790(2.033)$ & $-942.96056210(0.033)$ & -3013.8 \\
\hline $48-7$ & $-942.89286490(2.034)$ & $-942.91777010(0.169)$ & -2930.9 \\
\hline $48-8$ & $-942.84141260(2.035)$ & $-942.86387760(0.298)$ & -2838.5 \\
\hline $48-9$ & $-942.77675710(2.036)$ & $-942.79692110(0.421)$ & -2740.2 \\
\hline 48 & $-942.69655170(2.038)$ & $-942.71457160(0.534)$ & -2631.2 \\
\hline $58-8$ & $-942.81280280(2.024)$ & $-942.84670560(0.002)$ & -3679.9 \\
\hline $58-9$ & $-942.75868650(2.023)$ & $-942.78961490(0.144)$ & -3612.6 \\
\hline 58 & $-942.69754550(2.022)$ & $-942.72549300(0.272)$ & -3505.6 \\
\hline $60-2$ & $-943.01250760(2.035)$ & $-943.03458780(0.261)$ & -2731.7 \\
\hline $60-3$ & $-942.99161280(2.038)$ & $-943.00637890(0.596)$ & -2247.4 \\
\hline $60-4$ & $-942.95878990(2.040)$ & $-942.96827340(0.818)$ & -1703.3 \\
\hline $60-5$ & $-942.91429440(2.041)$ & $-942.92013910(0.952)$ & -1177.9 \\
\hline $60-6$ & $-942.85820830(2.041)$ & $-942.86169070(1.025)$ & -752.26 \\
\hline $60-7$ & $-942.79048280(2.040)$ & $-942.79254510(1.057)$ & -460.45 \\
\hline $60-8$ & $-942.71092450(2.038)$ & $-942.71222520(1.065)$ & -293.39 \\
\hline $60-9$ & $-942.61932280(2.037)$ & $-942.60296310(0.998)$ & 3455.8 \\
\hline 60 & $-942.51311180(2.018)$ & $-942.50635350(0.931)$ & 1364.1 \\
\hline $62-3$ & $-942.90675130(2.028)$ & $-942.93131050(0.189)$ & -2931.0 \\
\hline $62-4$ & $-942.81165370(2.028)$ & $-942.82847610(0.506)$ & -2425.8 \\
\hline $62-5$ & $-942.67957990(2.028)$ & $-942.69020750(0.733)$ & -1801.1 \\
\hline $62-6$ & $-942.50407460(2.027)$ & $-942.51019960(0.880)$ & -1172.0 \\
\hline $62-7$ & $-942.27708710(2.025)$ & $-942.26312770(0.218)$ & 1695.5 \\
\hline $62-8$ & $-941.98831900(2.023)$ & $-941.97074890(0.841)$ & 3262.4 \\
\hline $62-9$ & $-941.61545960(2.014)$ & $-941.60873720(1.012)$ & 1475.4 \\
\hline 62 & $-941.15992380(2.014)$ & $-941.15461330(0.867)$ & 1016.5 \\
\hline 64 & $-942.74012900(2.027)$ & $-942.77130410(0.006)$ & -3386.8 \\
\hline $65-9$ & $-942.59387730(2.016)$ & $-942.59992240(0.068)$ & -681.08 \\
\hline 65 & $-942.49158970(2.016)$ & $-942.48884070(0.602)$ & 426.70 \\
\hline $66-4$ & $-942.94911820(2.029)$ & $-942.97778730(0.024)$ & -3138.2 \\
\hline
\end{tabular}




\begin{tabular}{|c|c|c|c|}
\hline $66-5$ & $-942.90544590(2.029)$ & $-942.93028320(0.225)$ & -3021.7 \\
\hline $66-6$ & $-942.84961280(2.029)$ & $-942.87085460(0.401)$ & -2863.7 \\
\hline $66-7$ & $-942.78073750(2.029)$ & $-942.79868070(0.551)$ & -2664.5 \\
\hline $66-8$ & $-942.69784500(2.029)$ & $-942.71282080(0.675)$ & -2427.5 \\
\hline $66-9$ & $-942.59993000(2.029)$ & $-942.61228700(0.774)$ & -2161.0 \\
\hline 66 & $-942.48583570(2.028)$ & $-942.49591300(0.853)$ & -1881.2 \\
\hline $68-3$ & $-942.88995120(2.024)$ & $-942.91510130(0.256)$ & -3122.1 \\
\hline $68-4$ & $-942.77963370(2.023)$ & $-942.79807460(0.528)$ & -2707.2 \\
\hline $68-5$ & $-942.62563740(2.021)$ & $-942.63883000(0.715)$ & -2217.0 \\
\hline $68-6$ & $-942.41970260(2.020)$ & $-942.42900820(0.838)$ & -1727.9 \\
\hline $68-7$ & $-942.15140860(2.019)$ & $-942.15794510(0.918)$ & -1303.0 \\
\hline $68-8$ & $-941.80768220(2.018)$ & $-941.81231840(0.970)$ & -970.9 \\
\hline $68-9$ & $-941.37228040(2.017)$ & $-941.37568770(1.006)$ & -739.7 \\
\hline 68 & $-940.82519390(2.017)$ & $-940.82792550(1.034)$ & -609.9 \\
\hline $71-4$ & $-942.83846450(2.033)$ & $-942.86492600(0.318)$ & -3386.4 \\
\hline $71-8$ & $-942.18987040(2.032)$ & $-942.17823800(0.303)$ & 1476.6 \\
\hline $71-9$ & $-941.91814760(2.094)$ & $-941.90025710(0.579)$ & 2591.8 \\
\hline 71 & $-941.59136900(2.585)$ & $-941.59160560(1.593)$ & -52.4 \\
\hline $72-4$ & $-942.85002120(2.039)$ & $-942.88681820(0.167)$ & -4314.1 \\
\hline $72-5$ & $-942.76183010(2.031)$ & $-942.79258350(0.651)$ & -4891.0 \\
\hline $72-6$ & $-942.65225480(2.028)$ & $-942.67904610(1.057)$ & -6055.6 \\
\hline $72-7$ & $-942.51970810(2.026)$ & $-942.54487440(1.371)$ & -8432.6 \\
\hline $72-8$ & $-942.36180800(2.023)$ & $-942.38768570(1.600)$ & -13426.7 \\
\hline $72-9$ & $-942.17596940(2.021)$ & $-942.20449820(1.760)$ & -23989.8 \\
\hline 72 & $-941.94283500(2.141)$ & $-941.99175530(1.868)$ & -39388.1 \\
\hline $73-4$ & $-942.78329870(2.065)$ & $-942.80890690(0.208)$ & -3026.6 \\
\hline $73-5$ & $-942.64296610(2.063)$ & $-942.66157040(0.569)$ & -2733.0 \\
\hline $73-6$ & $-942.46176270(2.055)$ & $-942.47437540(0.837)$ & -2272.7 \\
\hline $73-7$ & $-942.23294460(2.045)$ & $-942.24098110(0.998)$ & -1684.6 \\
\hline $73-8$ & $-941.94829950(2.037)$ & $-941.95315160(1.070)$ & -1101.3 \\
\hline $73-9$ & $-941.59795960(2.030)$ & $-941.60076500(1.086)$ & -652.2 \\
\hline 73 & $-941.17031920(2.026)$ & $-941.17189780(1.077)$ & -365.2 \\
\hline $74-4$ & $-942.80048690(2.070)$ & $-942.83408380(0.016)$ & -3589.9 \\
\hline $74-5$ & $-942.66771300(2.108)$ & $-942.69706220(0.369)$ & -3704.1 \\
\hline $74-6$ & $-942.49340310(2.173)$ & $-942.51890210(0.710)$ & -3825.3 \\
\hline $74-7$ & $-942.26899320(2.274)$ & $-942.29131410(1.018)$ & -3900.4 \\
\hline $74-8$ & $-941.98315710(2.406)$ & $-941.99303110(0.686)$ & -1259.9 \\
\hline $74-9$ & $-941.62587040(2.764)$ & $-941.61860490(0.411)$ & 677.7 \\
\hline 74 & $-941.16259020(2.730)$ & $-941.15874060(1.278)$ & 581.9 \\
\hline $75-7$ & $-942.27472010(2.056)$ & $-942.31118600(0.134)$ & -4164.1 \\
\hline 75 & $-941.20864000(2.133)$ & $-941.22084730(0.160)$ & -1357.7 \\
\hline
\end{tabular}


Table S6. Detailed vibrational modes HAP of positive distortion and the corresponding geometrical characters, HOMO-LUMO gaps (H-L gap, eV) of the closed-shell singlet states, T-S gaps between the closed-shell singlet and triplet states (E(triplet)-E(singlet), $\mathrm{kcal} / \mathrm{mol})$, and $\left\langle\mathrm{S}^{2}\right\rangle$ values, SOMO $(\alpha, \beta)$, spin densities of the OS states of the mode-distorted HAP configurations.

\begin{tabular}{|c|c|c|c|c|c|}
\hline $\begin{array}{l}\text { Modes } \\
\mathrm{S}^{2}\end{array}$ & $\begin{array}{c}\text { HO-LU gap } \\
\text { (ev) } \\
\text { T-S gap } \\
\text { (kcal/mol) }\end{array}$ & $\begin{array}{c}\text { Distortion } \\
\text { Modes }\end{array}$ & $\begin{array}{l}\text { HOMO or SOMO } \\
(\alpha)\end{array}$ & $\begin{array}{c}\text { LUMO or SOMO } \\
(\beta)\end{array}$ & Spin density \\
\hline $\begin{array}{l}17-1 \\
0.00\end{array}$ & $\begin{array}{c}2.34 \\
25.99\end{array}$ & & & & \\
\hline $\begin{array}{l}17-2 \\
0.00\end{array}$ & $\begin{array}{c}2.34 \\
25.56\end{array}$ & & & & \\
\hline $\begin{array}{l}17-3 \\
0.00\end{array}$ & $\begin{array}{l}2.285 \\
24.87\end{array}$ & & & & \\
\hline $\begin{array}{l}17-4 \\
0.00\end{array}$ & $\begin{array}{l}2.258 \\
23.99\end{array}$ & & & & \\
\hline $\begin{array}{l}17-5 \\
0.00\end{array}$ & $\begin{array}{l}2.204 \\
22.86\end{array}$ & & & & \\
\hline $\begin{array}{l}17-6 \\
0.00\end{array}$ & $\begin{array}{l}2.176 \\
21.48\end{array}$ & & & & \\
\hline & $\begin{array}{l}2.095 \\
19.92\end{array}$ & & & & \\
\hline $\begin{array}{l}17-8 \\
0.0272\end{array}$ & $\begin{array}{c}2.04 \\
18.10\end{array}$ & & & & \\
\hline
\end{tabular}




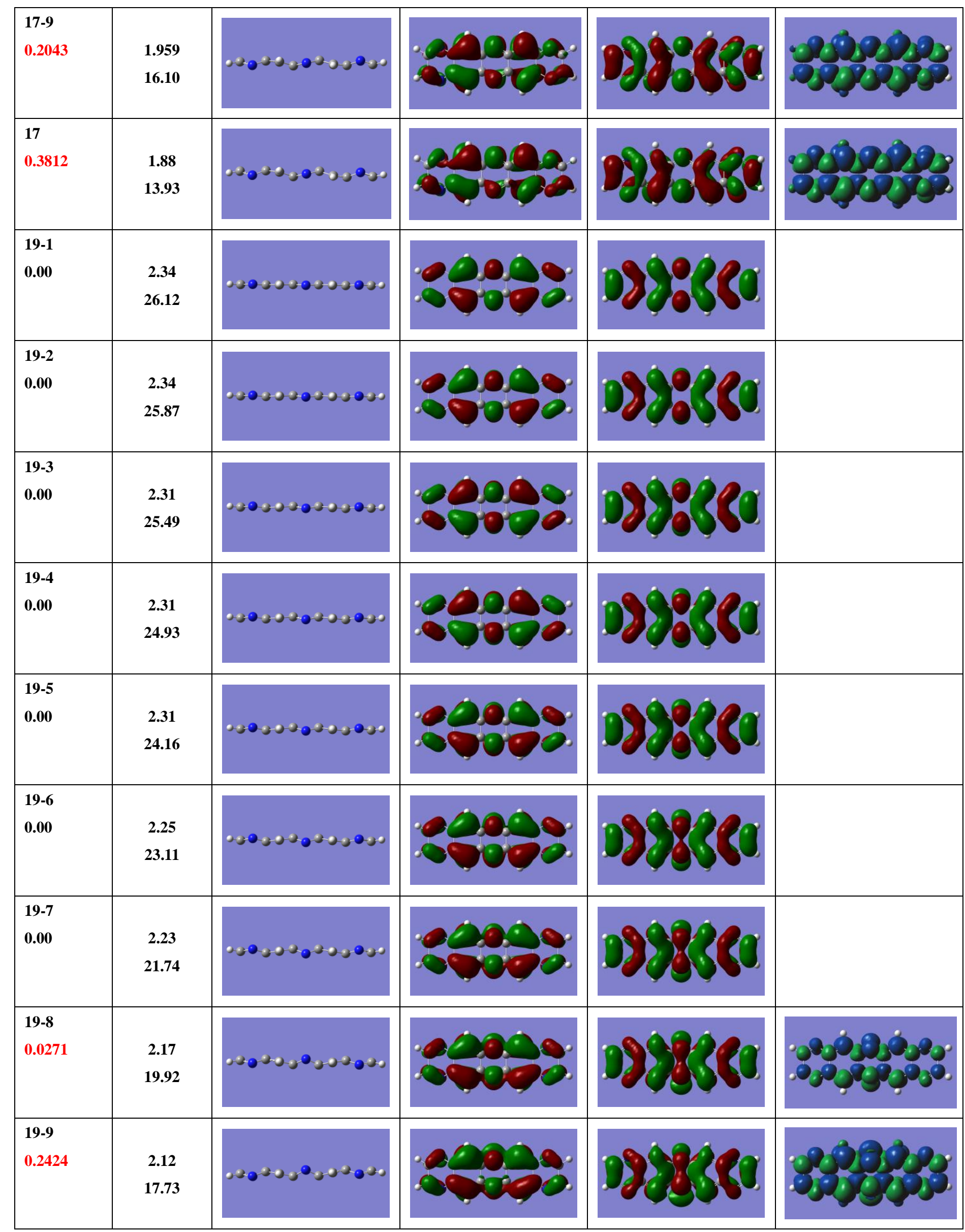




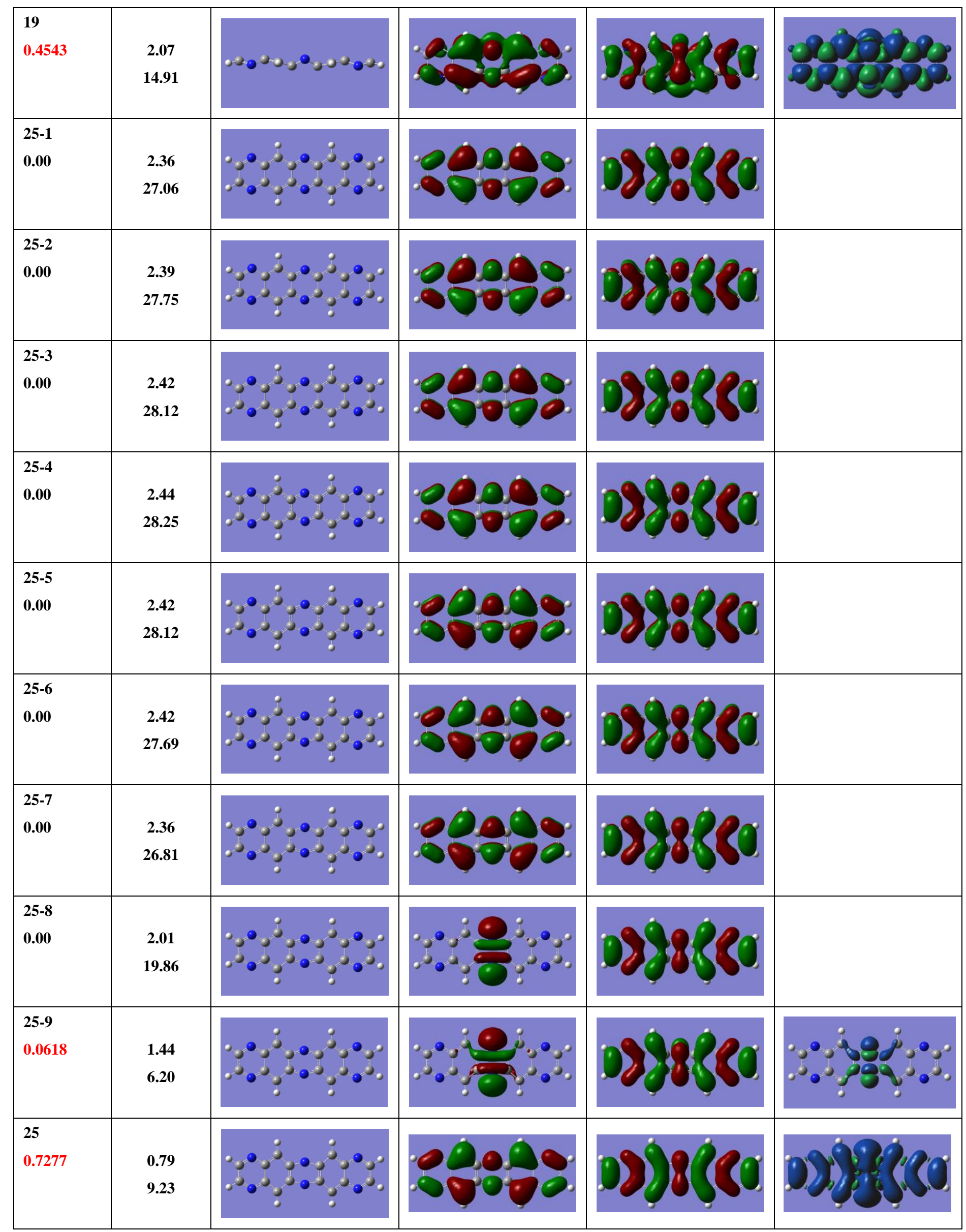




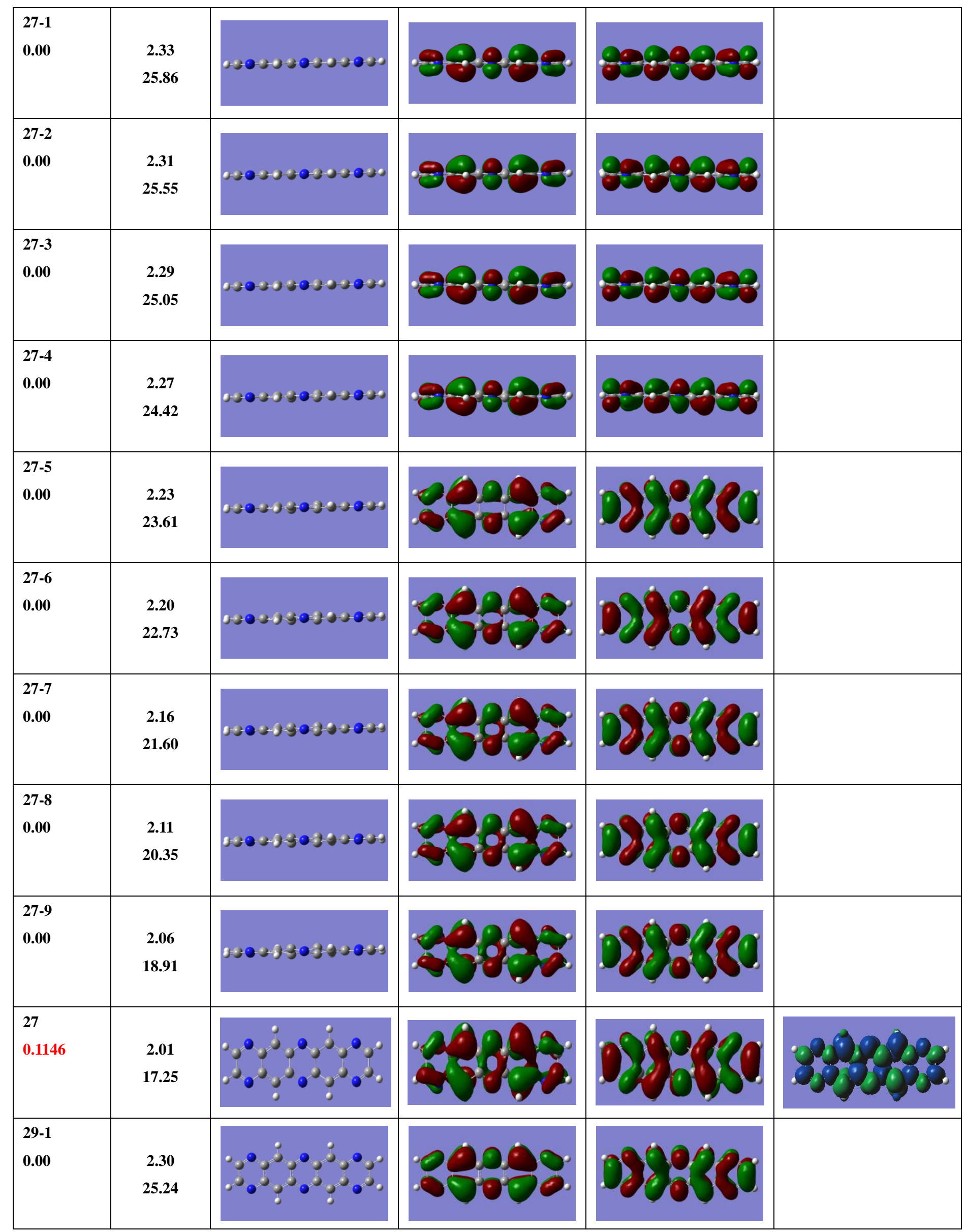




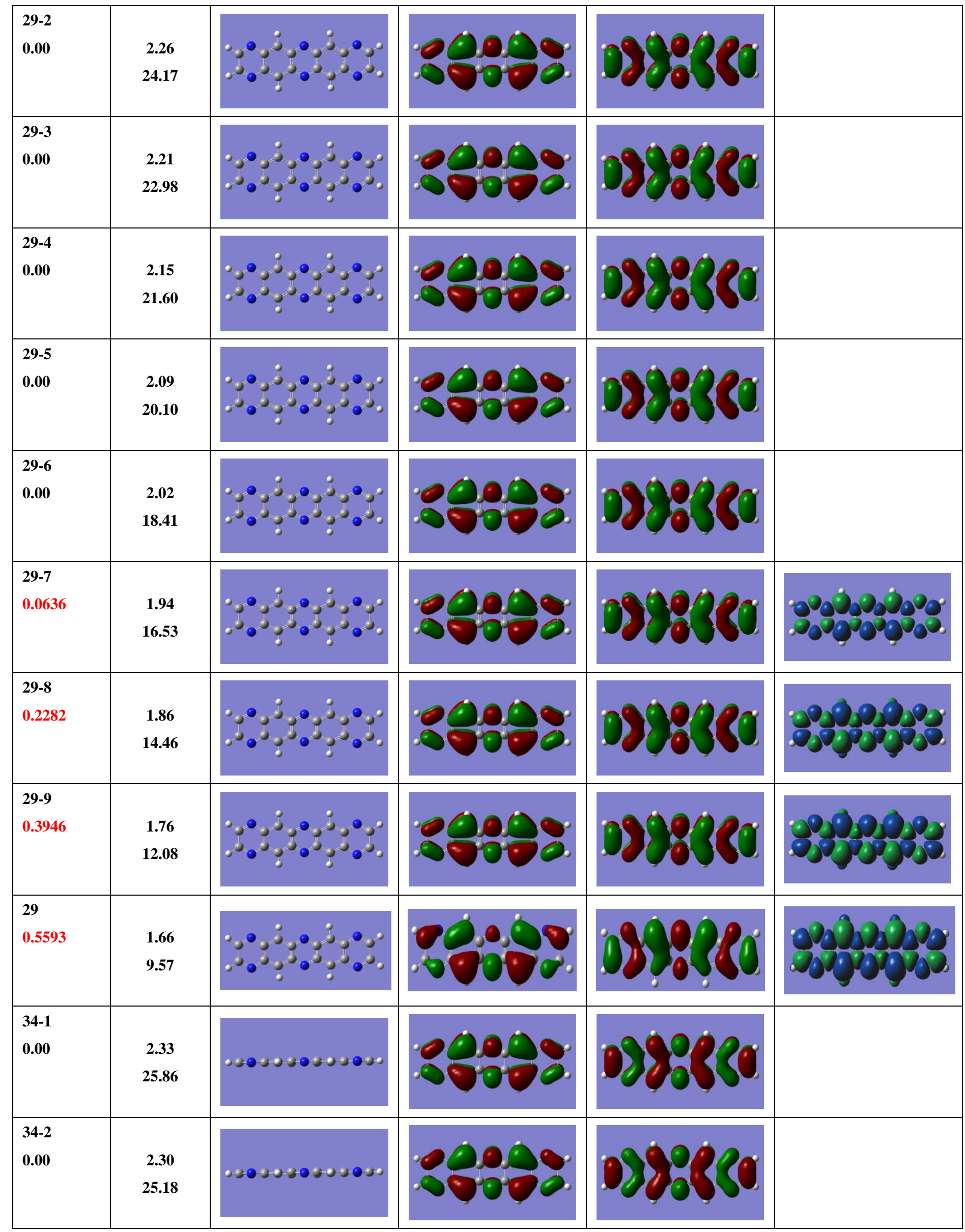




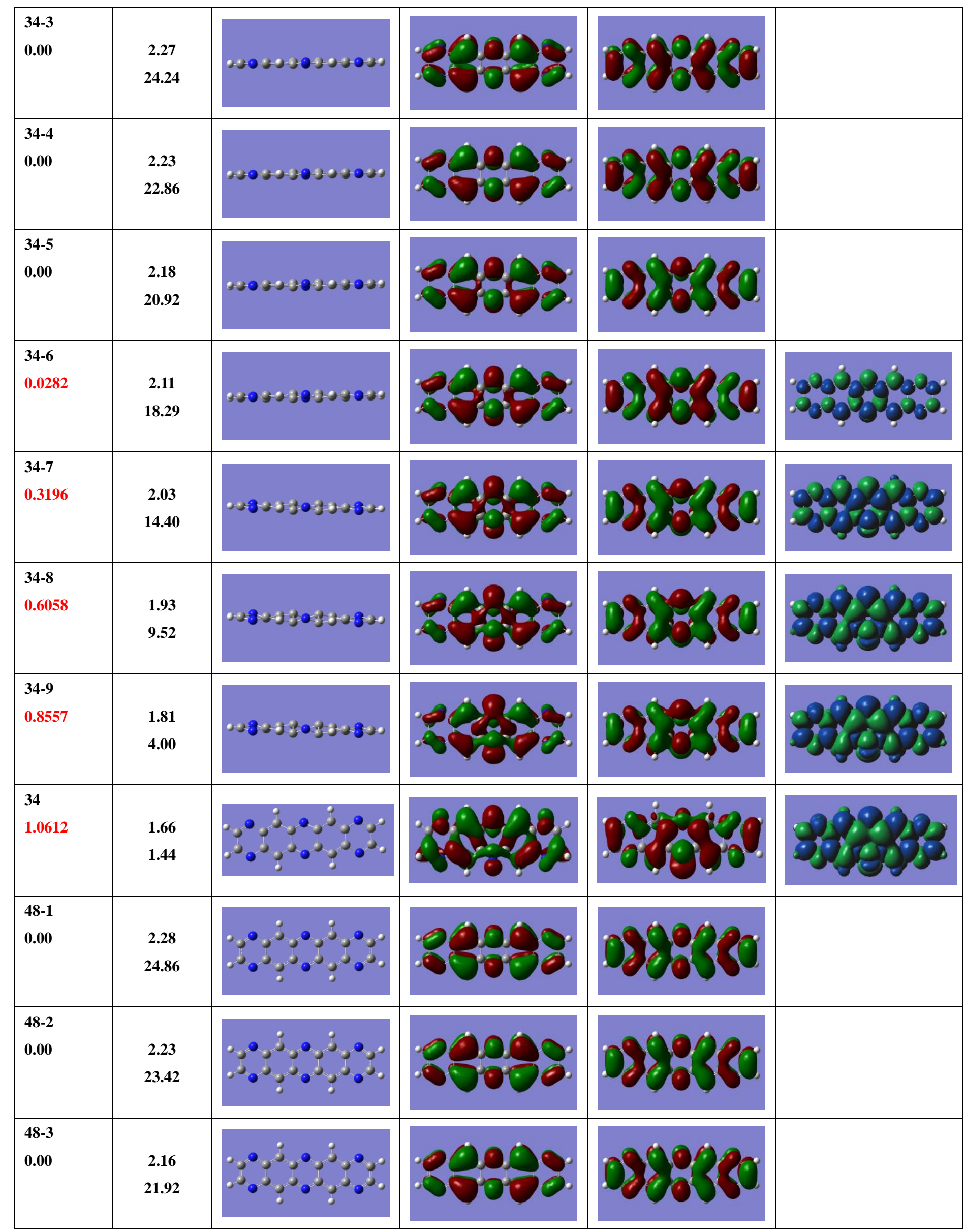




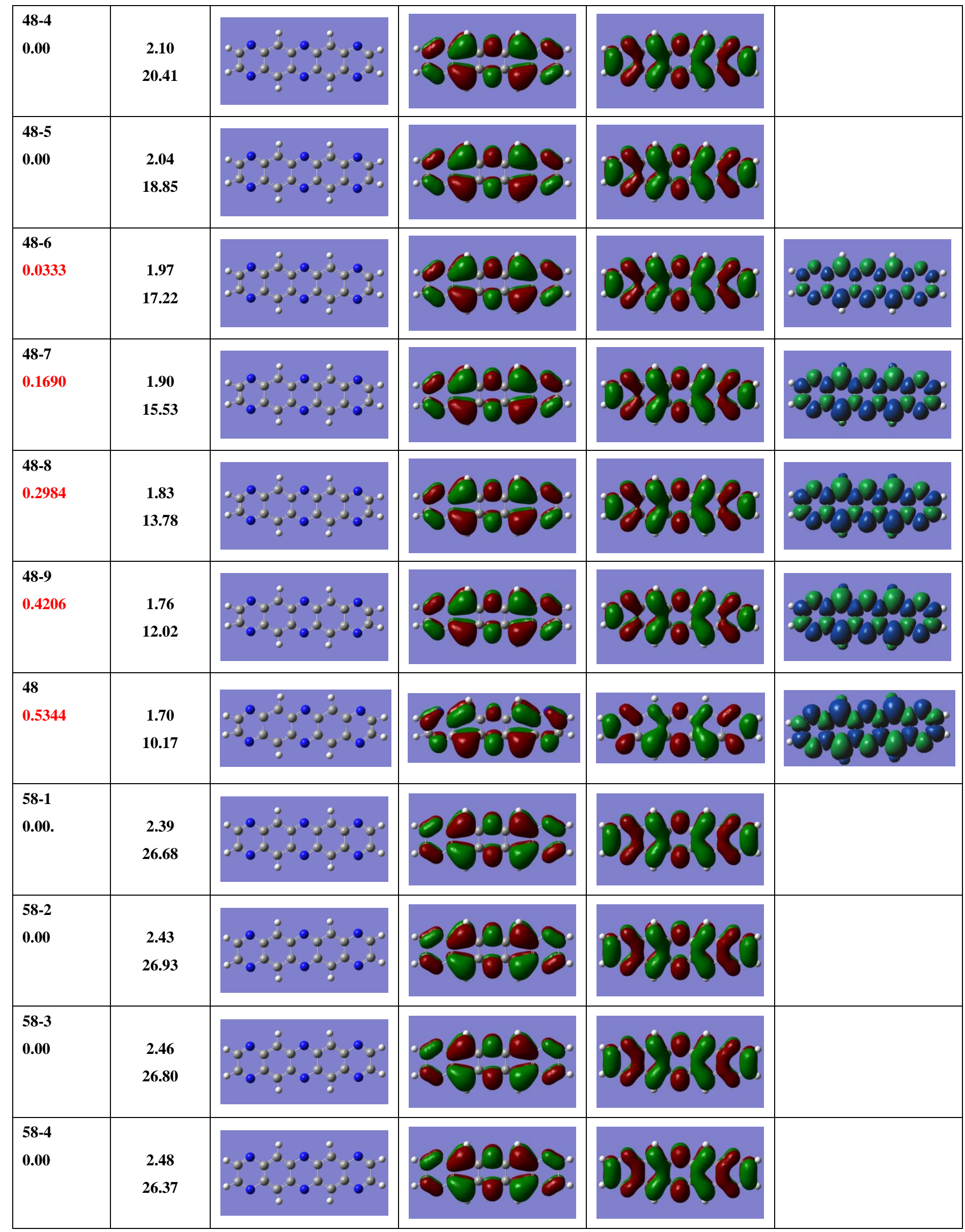




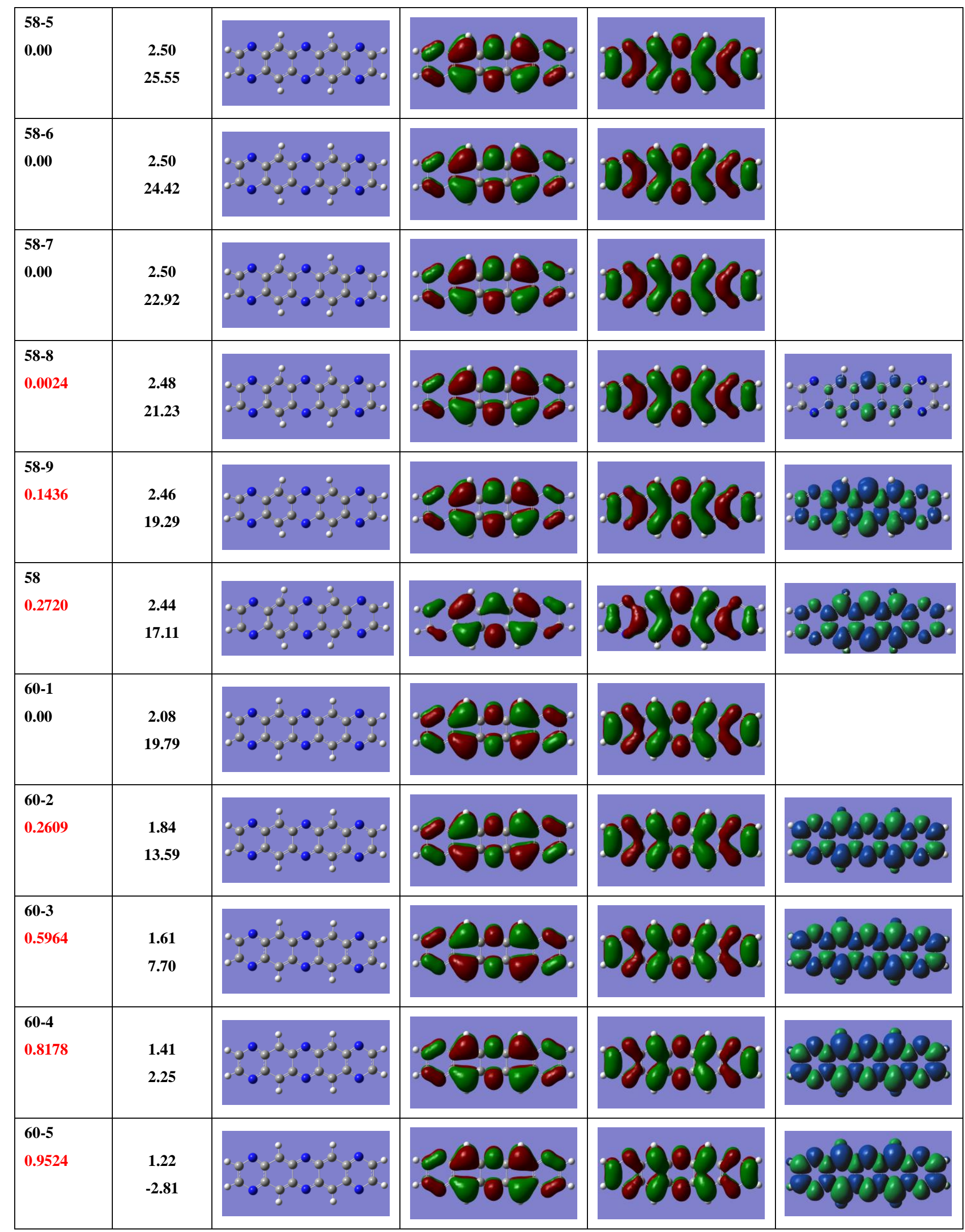




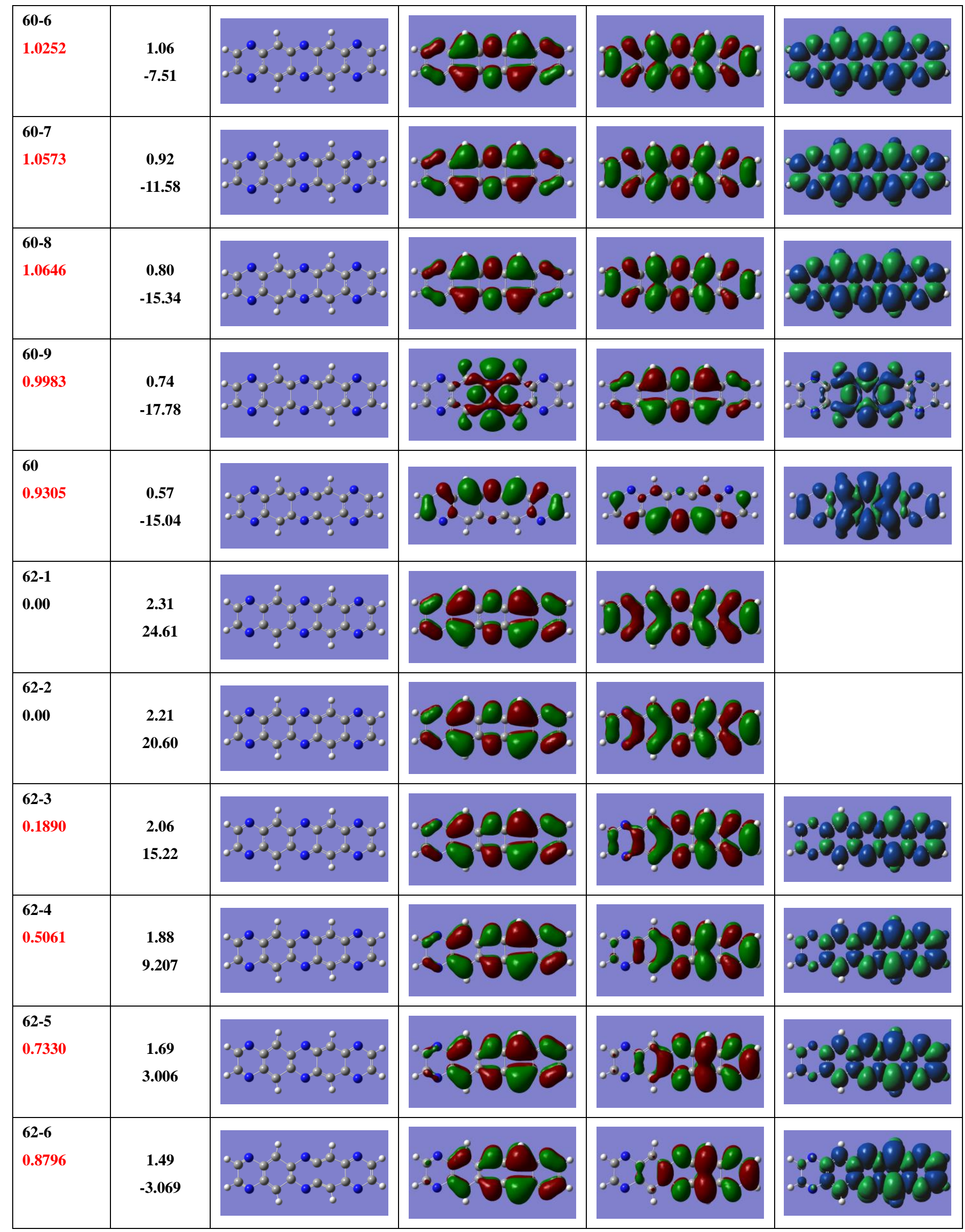




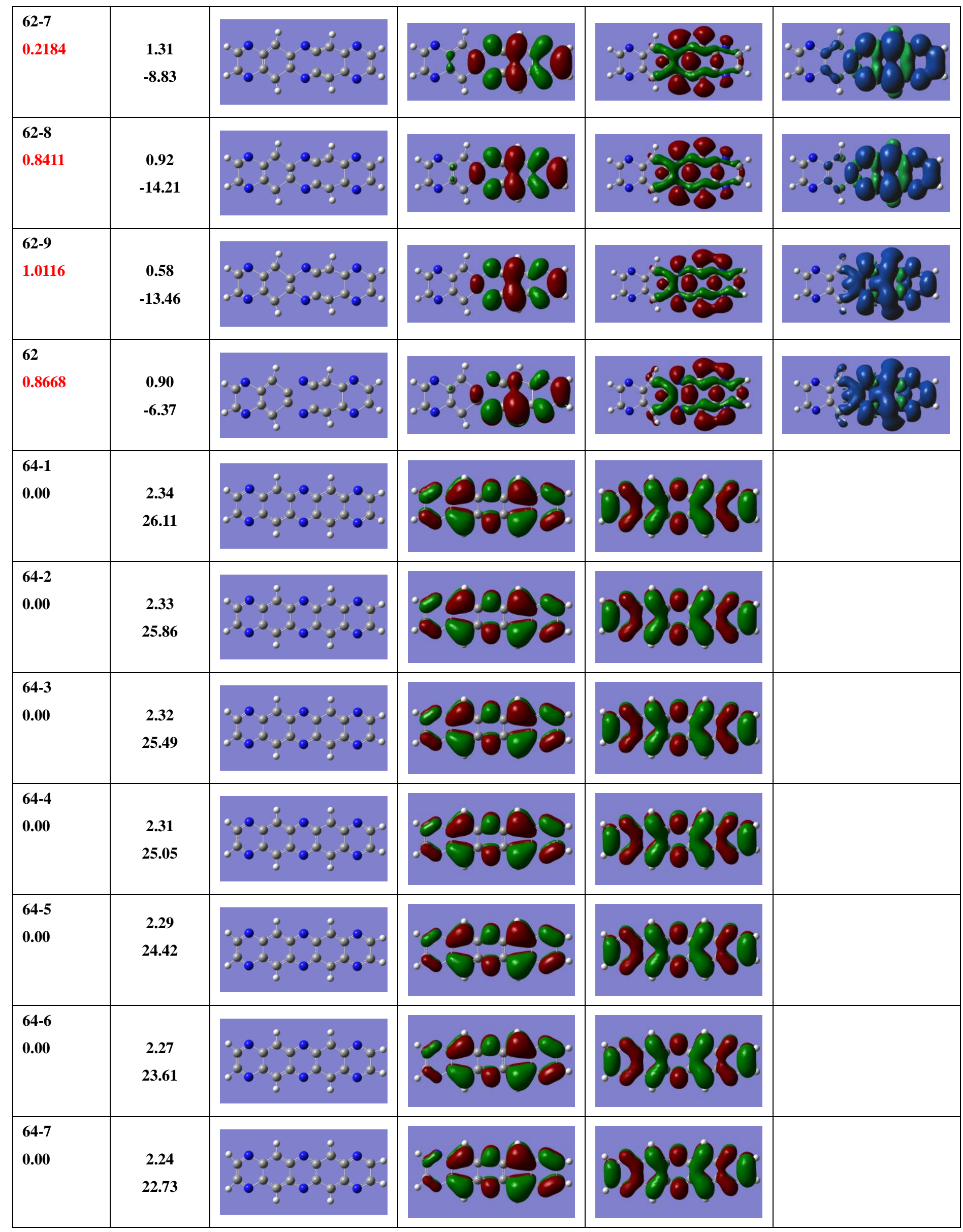




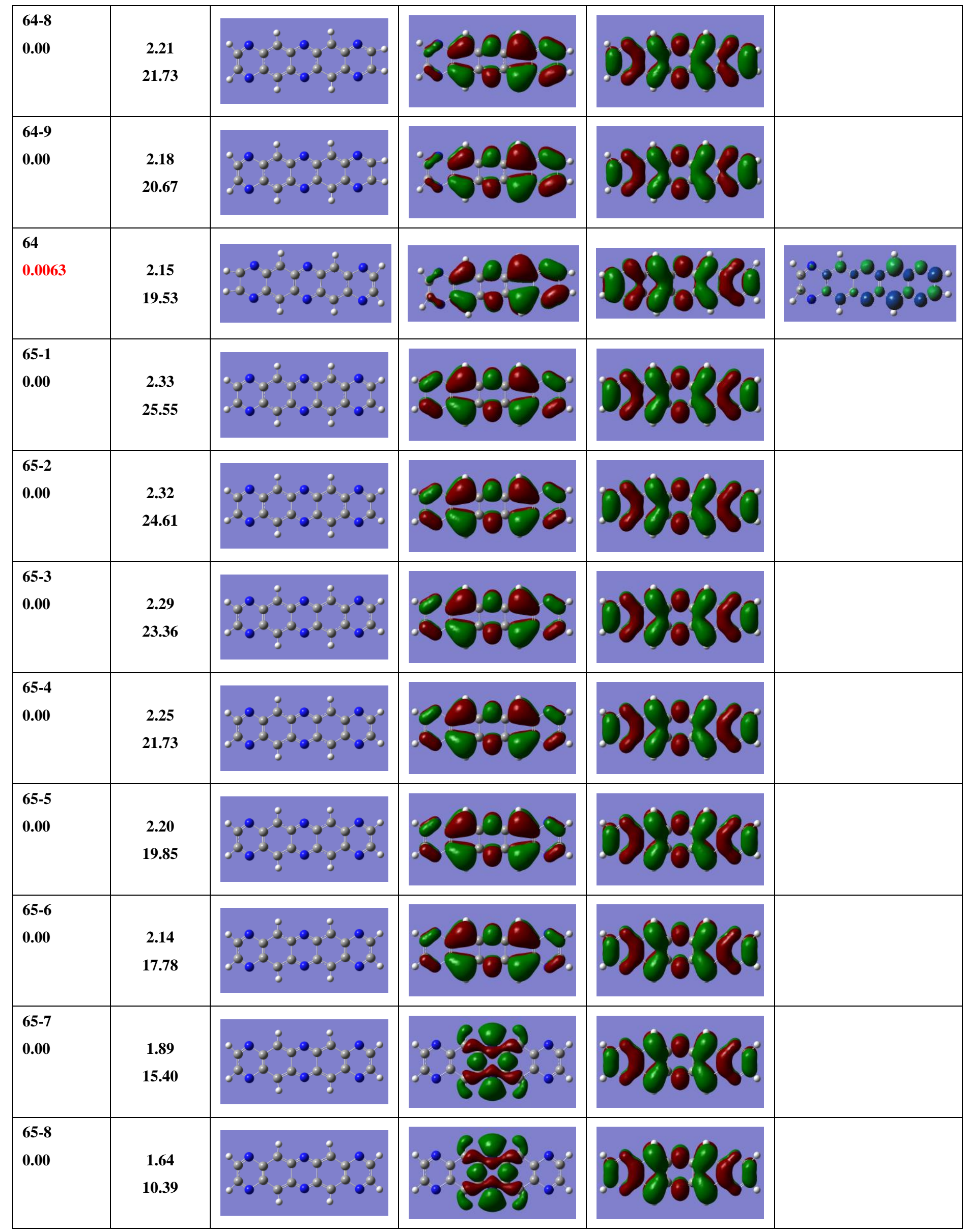




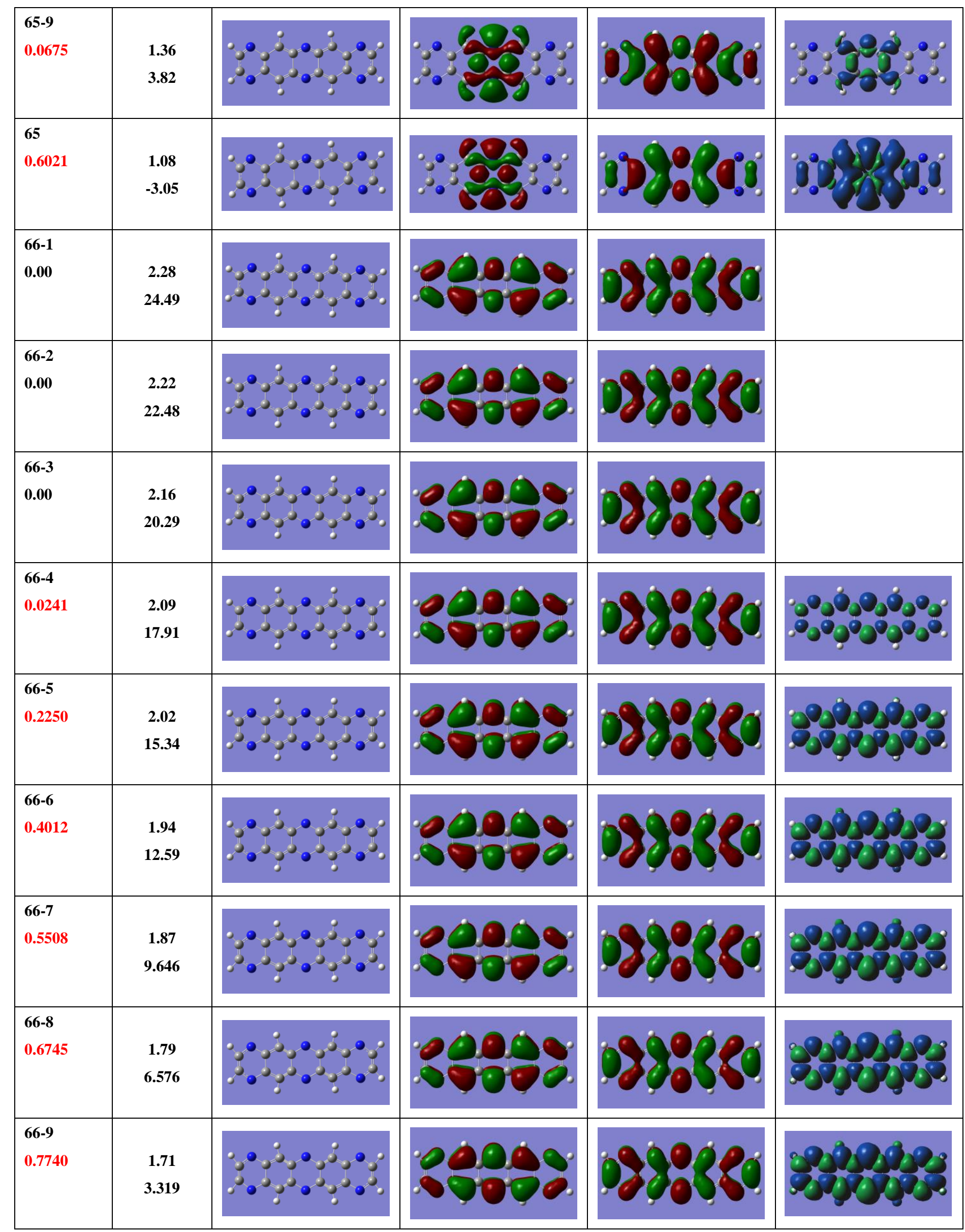




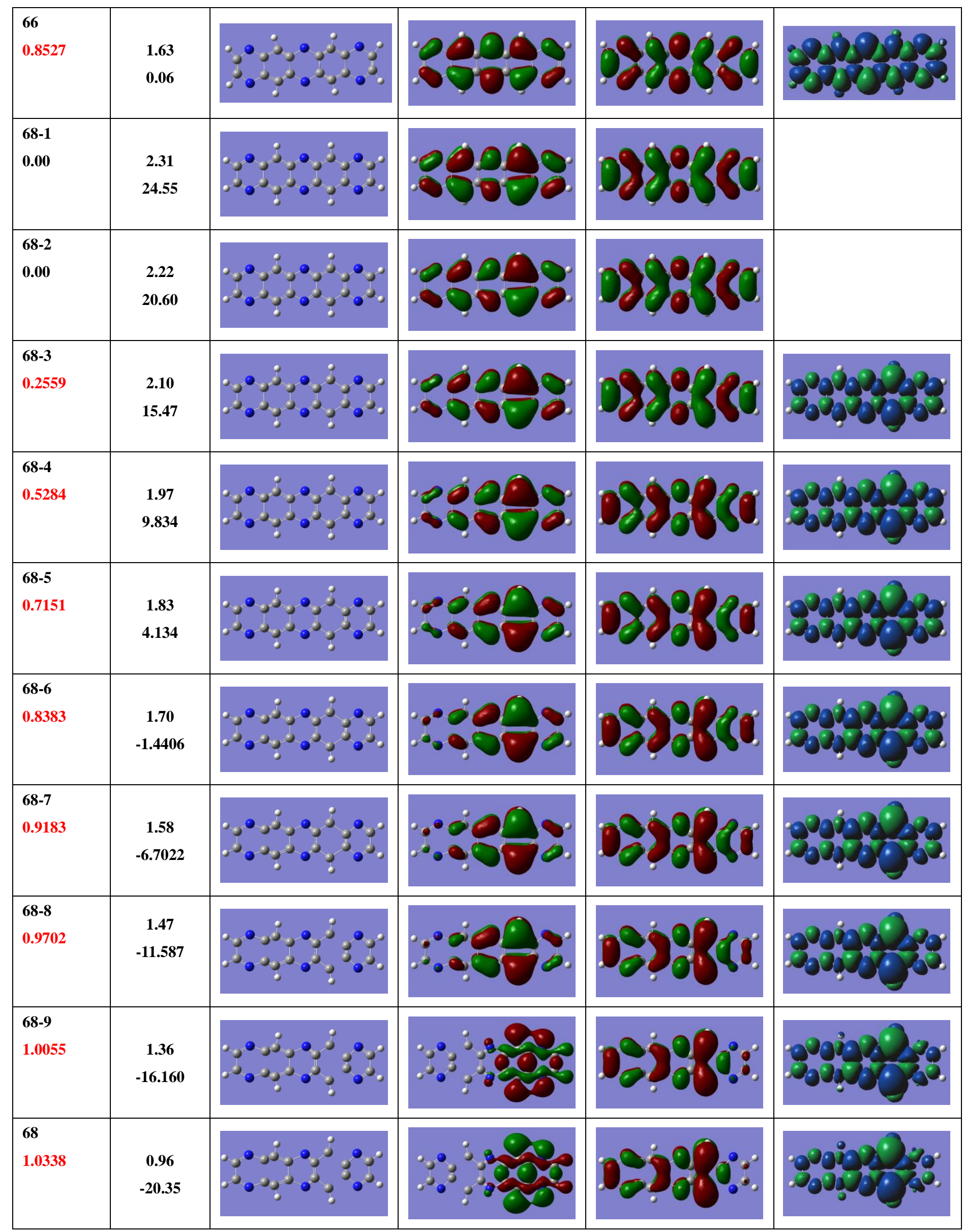




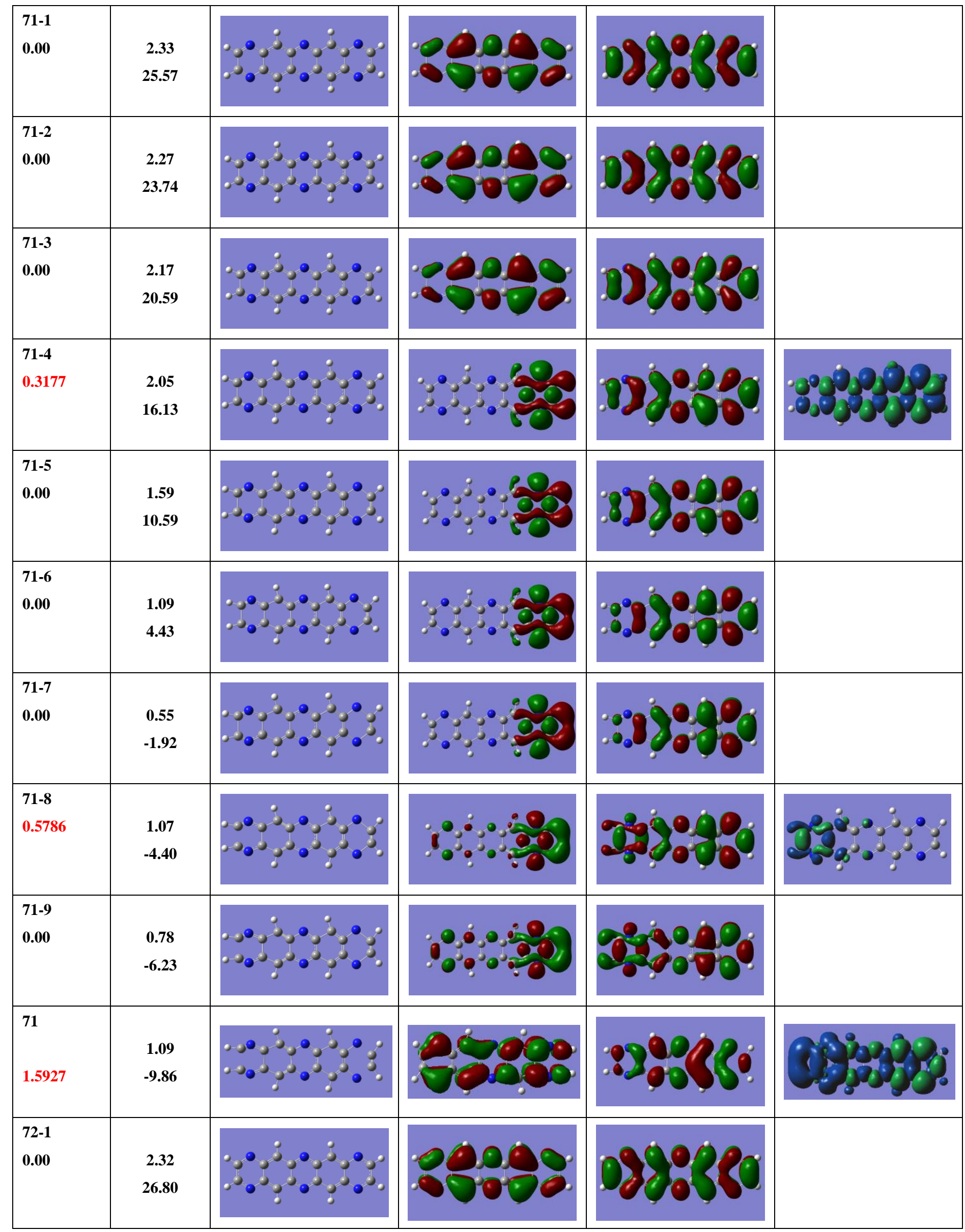




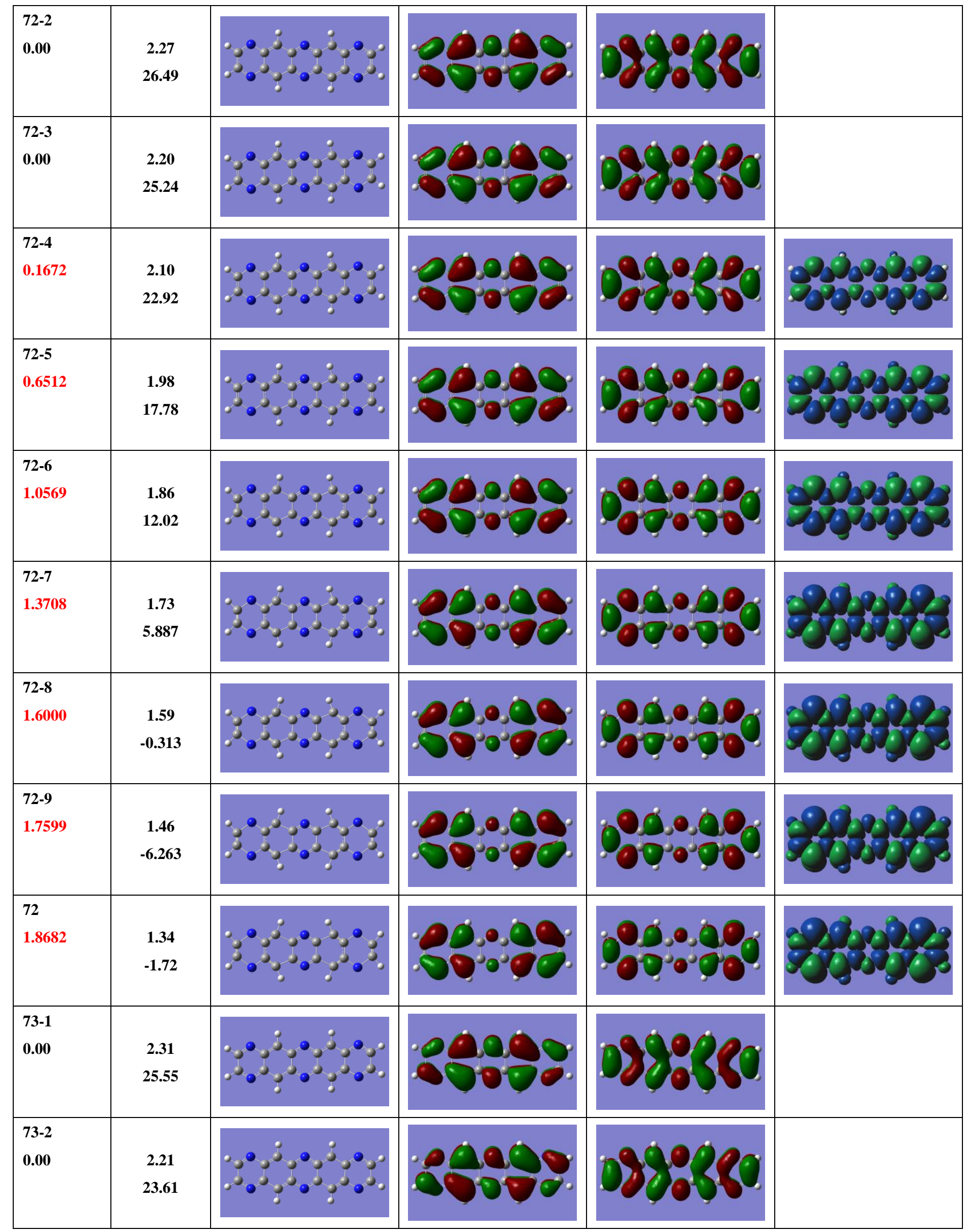




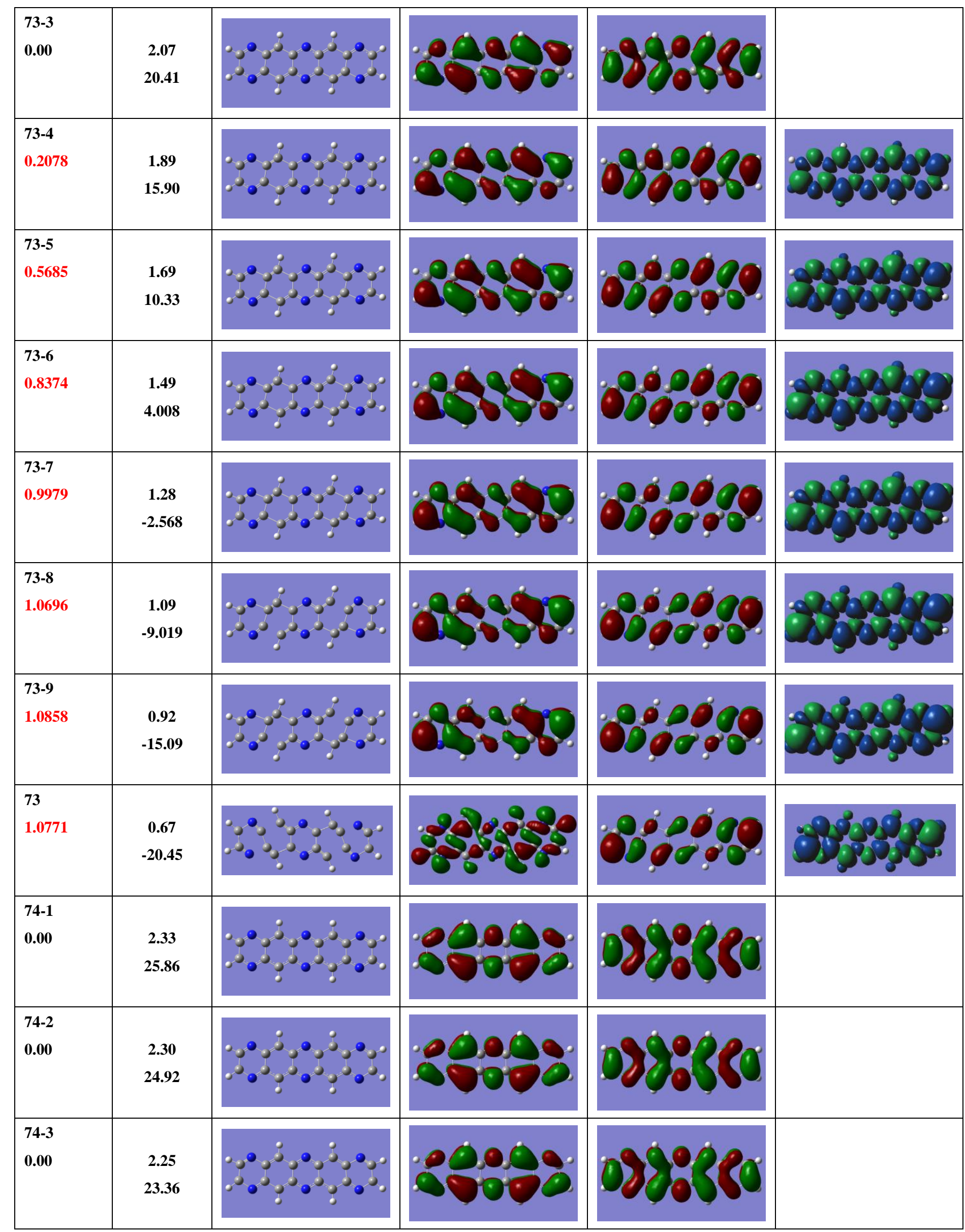




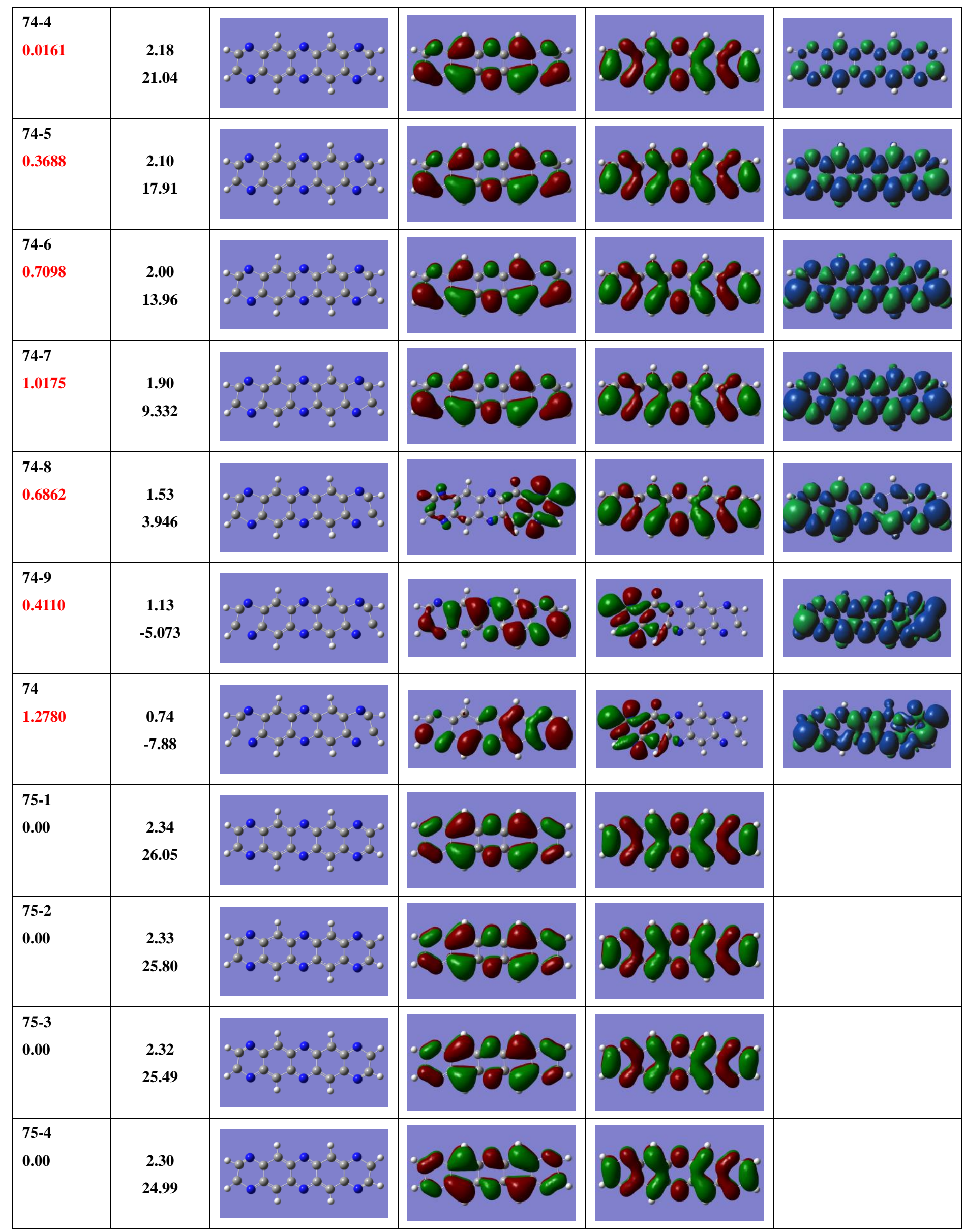




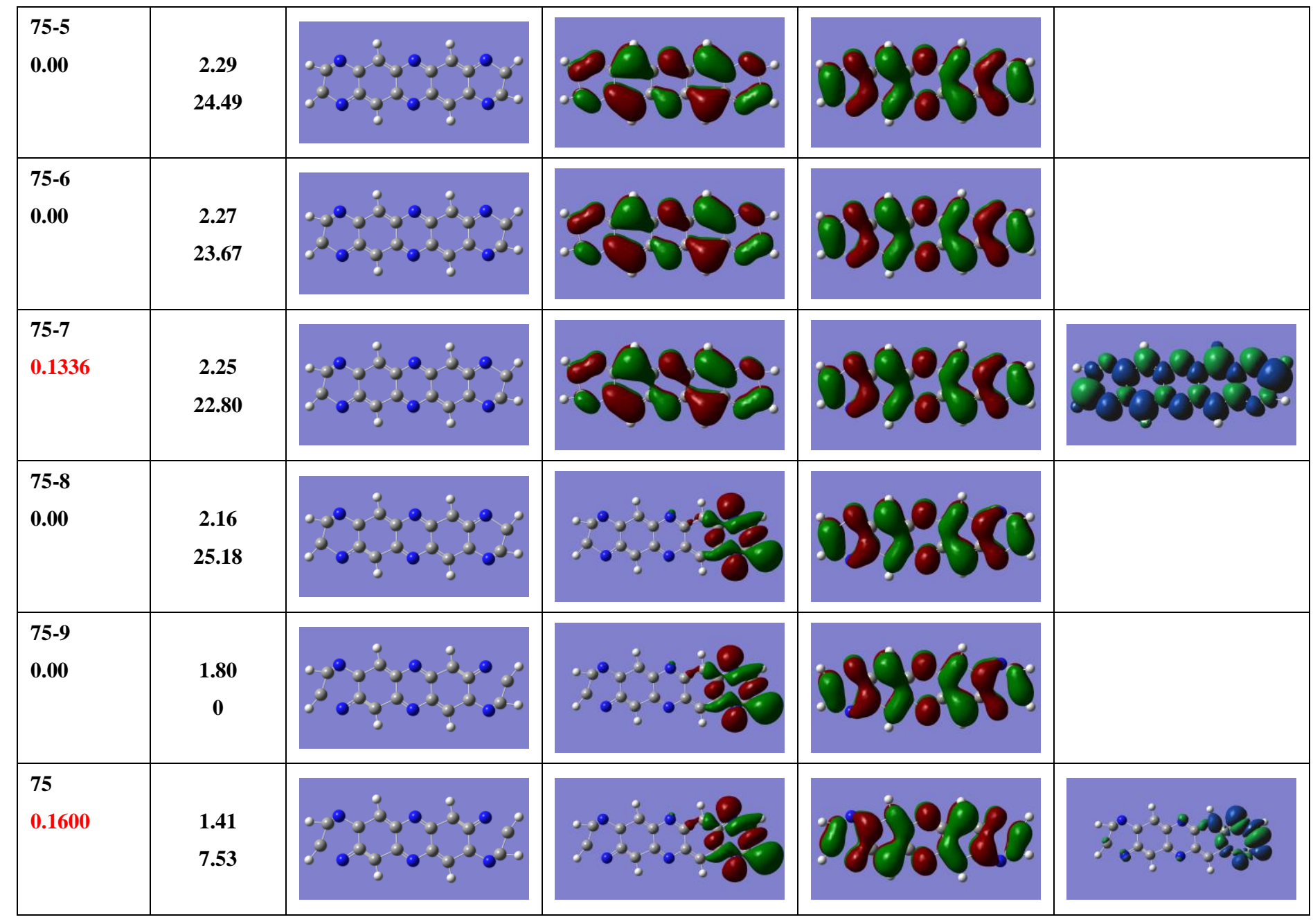

Table S7. The cross-linking C-C bond lengths of the distorted configurations according to 19 diradical vibrational modes.

\begin{tabular}{|c|c|}
\hline 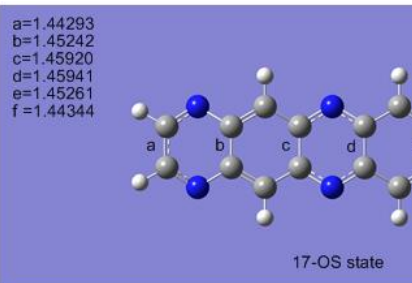 & 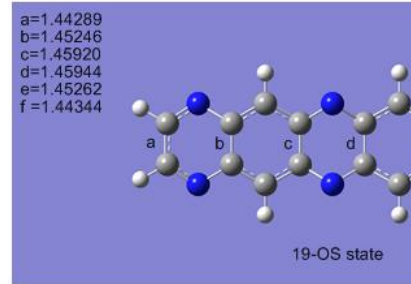 \\
\hline 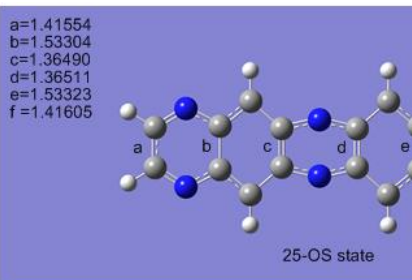 & 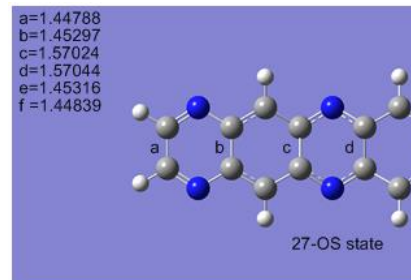 \\
\hline
\end{tabular}




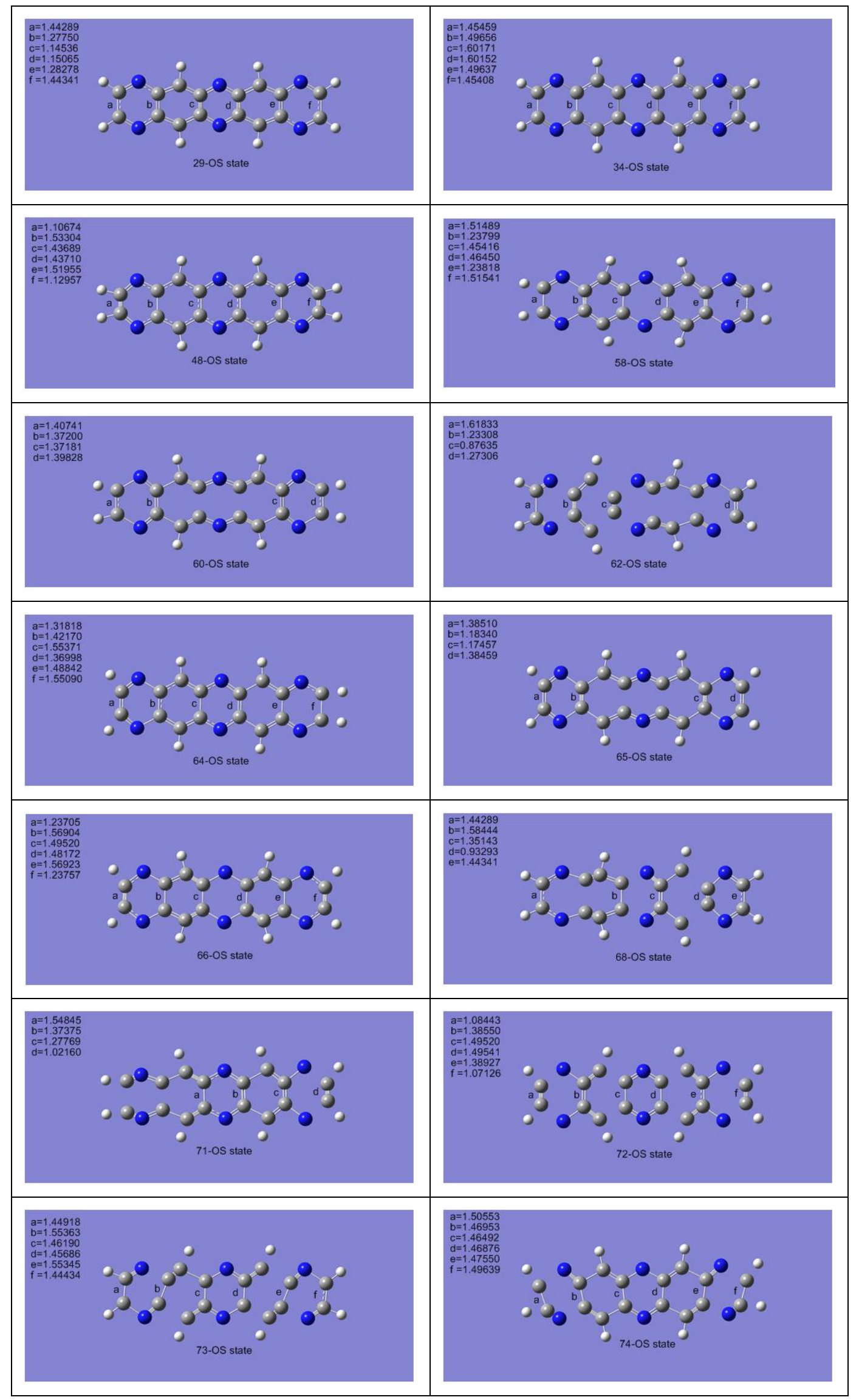




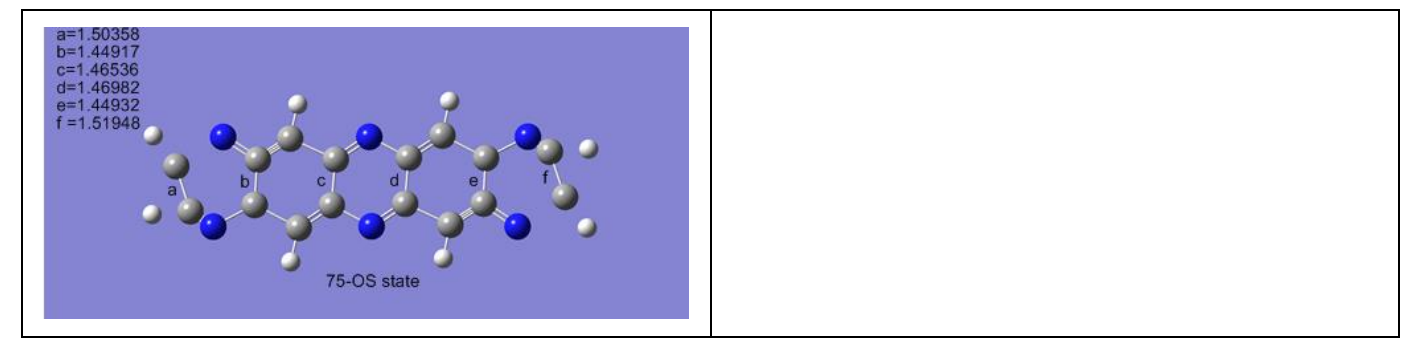

\section{The Complete Citation for Ref. 60}

Frisch, M. J.; Trucks, G. W.; Schlegel, H. B.; Scuseria, G. E.; Robb, M. A.; Cheeseman, J. R.; Montgomery, J. A., Jr; ; Vreven, T.; Kudin, K. N.; Burant, J. C.; Millam, J. M.; Iyengar, S. S.; Tomasi, J.; Barone, V.; Mennucci, B.; Cossi, M.; Scalmani, G.; Rega, N.; Petersson, G. A.; Nakatsuji, H.; Hada, M.; Ehara, M.; Toyota, M.; Fukuda, R.; Hasegawa, J.; Ishida, M.; Nakajima, T.; Honda, Y.; Kitao, O.; Nakai, H.; Klene, M.; Li, X.; Knox, J. E.; Hratchian, H. P.; Cross, J. B.; Adamo, C.; Jaramillo, J.; Gomperts, R.; Stratmann, R. E.; Yazyev, O.; Austin, A. J.; Cammi, R.; Pomelli, C.; Ochterski, J. W.; Ayala, P. Y.; Morokuma, K.; Voth, G. A.; Salvador, P.; Dannenberg, J. J.; Zakrzewski, V. G.; Dapprich, S.; Daniels, A. D.; Strain, M. C.; Farkas, O.; Malick, D. K.; Rabuck, A. D.; Raghavachari, K.; Foresman, J. B.; Ortiz, J. V.; Cui, Q.; Baboul, A. G.; Clifford, S.; Cioslowski, J.; Stefanov, B. B.; Liu, G.; Liashenko, A.; Piskorz, P.; Komaromi, I.; Martin, R. L.; Fox, D. J.; Keith, T.; Al-Laham, M. A.; Peng, C. Y.; Nanayakkara, A.; Challacombe, M.; Gill, P. M. W.; Johnson, B.; Chen, W.; Wong, M. W.; Gonzalez, C.; Pople, J. A. Gaussian 03; Gaussian, Inc.: Wallingford, CT, 2004. 